\title{
ON THE EXISTENCE AND UNIQUENESS OF POSITIVE SOLUTIONS FOR COMPETING SPECIES MODELS WITH DIFFUSION
}

\author{
E. N. DANCER
}

\begin{abstract}
In this paper, we consider strictly positive solutions of competing species systems with diffusion under Dirichlet boundary conditions. We obtain a good understanding of when strictly positive solutions exist, obtain new nonuniqueness results and a number of other results, showing how complicated these equations can be. In particular, we consider how the shape of the underlying domain affects the behaviour of the equations.
\end{abstract}

The purpose of this paper is to obtain much better results on the existence and uniqueness of strictly positive stationary (that is time-independent) solutions of

$$
\begin{aligned}
L \frac{\partial u}{\partial t} & =\Delta u+u(a-u-c v) \\
\frac{\partial v}{\partial t} & =\Delta v+v(d-v-e u) \quad \text { in } \Omega \times R \\
u & =v=0 \quad \text { on } \partial \Omega \times R .
\end{aligned}
$$

Here $a, c, d, e, L>0$ and $\Omega$ is a bounded open set in $R^{m}$. Here $u=$ $u(x, t)$. Note that we could replace $-u$ by $-\alpha u$ where $\alpha>0$ in the first equation, $-v$ by $-\beta v$ in the second equation where $\beta>0$, and $\frac{\partial v}{\partial t}$ by $L_{2} \frac{\partial v}{\partial t}$ where $L_{2}>0$. However, these could be removed by simple rescalings and hence we have not lost generality.

The above equations are of competing species type (with diffusion). Here $u, v$ usually represent the population of species. Thus it is natural to look for nonnegative solutions.

For stationary solutions, the main point of interest is the existence and uniqueness of strictly positive solutions, that is solutions with both components strictly positive in $\Omega$. This problem has been studied extensively in $[4,5,6$, $8,9,24]$ where many further references can be found. However, the results are far from complete.

Let $\bar{u}$ denote the maximal solution of the first equation when $v$ vanishes identically and $u=0$ on $\partial \Omega$. We define $\bar{v}$ analogously. We assume that

Received by the editors July 31, 1989.

1980 Mathematics Subject Classification (1985 Revision). Primary 35J55; Secondary 47H15, $92 \mathrm{~A} 15$.

Key words and phrases. Competing species systems, strictly positive solutions, uniqueness, domain perturbation. 
$a, d>\lambda_{1}$ where $\lambda_{1}$ denotes the first eigenvalue of $-\Delta$ on $\partial \Omega$ (for our boundary conditions).

If $(\bar{u}, 0)$ and $(0, \bar{v})$ are both unstable or both stable for the parabolic system and a weak nondegeneracy assumption holds, we proved in [8] that there is a strictly positive solution. Hence the case of interest is when exactly one of $(\bar{u}, 0)$ and $(0, \bar{v})$ is stable. We showed in [9] that in this case there may or may not be a strictly positive solution. Here we obtain a good geometric understanding for which parameters there is a strictly positive solution. We show that, in the interior of the set $T$ where there is a strictly positive solution while exactly one of $(\bar{u}, 0)$ and $(0, \bar{v})$ is stable, there are at least two strictly positive solutions. One must be an asymptotically stable solution and another an unstable solution. We also prove that for almost all pairs $(a, d)$ (with $\left.a, d>\lambda_{1}\right)$ there is an open set of pairs $(c, e)$ in $R^{2}$ for which the above assumptions are valid. This is an interesting contrast to [6] where it is shown when $a=d$ there can only be strictly positive solutions when $(\bar{u}, 0)$ and $(0, \bar{v})$ are both stable or both unstable and with the Neumann problem where $T$ is always empty. We also prove some estimates of the extent of the set $T$. It turns out that $T$ naturally splits into two parts $T^{+}$and $T^{-}$. The above results answer a question in [24].

We also obtain examples of star-shaped sets $\Omega$ where the two solutions $(\bar{u}, 0)$ and $(0, \bar{v})$ are both unstable and nondegenerate (and $\left.a, d>\lambda_{1}\right)$ while the system has more than one strictly positive solution. This appears to be the first such example. We present examples of such nonuniqueness in two parameter ranges. One is where $d$ is close to $a$ while the second is where $a$ is fixed and $d$ is very large. The first is interesting because it is known [6] that if $d=a$, then uniqueness holds if $(\bar{u}, 0)$ and $(0, \bar{v})$ are both unstable. Both examples depend upon the domain perturbation results in [14]. We do not know if this nonuniqueness holds if $m=1$ or if $\Omega$ is convex.

We also produce a number of other counterexamples which show how complicated this simple looking equation is. Indeed, most of the questions one might ask turn out to have negative answers. One of these answers a question in [6].

While our main emphasis is on the stationary solutions, our methods have implications for the asymptotic behaviour of time dependent solutions. We discuss this fairly briefly.

One of the advantages of our methods is that they are quite flexible. For example, most of our results continue to hold for many other (including mixed) boundary conditions, for rather more general nonlinearities if we replace $-\Delta$ by a second order linear (not necessarily selfadjoint) operator and if $-\Delta$ is replaced by two different such operators in the two equations.

In $\S 1$, we obtain some basic results on the geometry of the solution set while in $\S 2$ we obtain conditions on when the solution set is other than the obvious set. In $\S 3$ we combine our earlier ideas with domain perturbation results to discuss uniqueness and to construct some other counterexamples, while in $\S 4$ we briefly discuss the time-dependent problem. 


\section{THE GEOMETRY OF THE SOLUTION SET}

We consider nonnegative stationary solutions of (1); that is, nonnegative solutions of

$$
\begin{aligned}
-\Delta u & =u(a-u-c v) \\
-\Delta v & =v(d-v-e u) \quad \text { in } \Omega, \\
u & =v=0 \quad \text { on } \partial \Omega .
\end{aligned}
$$

For simplicity, we assume that $\Omega$ is smooth. By solutions of (2) we will mean elements of $W^{1,2}(\Omega) \cap C_{0}(\bar{\Omega})$, where $C_{0}(\bar{\Omega})$ means the continuous functions on $\bar{\Omega}$ vanishing on $\partial \Omega$ with the usual norm. (In fact, at the expense of a little effort, this regularity assumption on $\Omega$ can be entirely removed by looking for solutions in $L^{\infty}(\Omega) \cap \dot{W}^{1,2}(\Omega)$ and by writing our equation as an equation on $L^{p}(\Omega) \oplus L^{p}(\Omega)$ for suitable large $p$.) Here we will use $C_{0}(\bar{\Omega}) \oplus C_{0}(\bar{\Omega})$ as our basic space.

First, as in [8], we see that, if $a \leq \lambda_{1}$, all nonnegative solutions have $u \equiv 0$. Here $\lambda_{1}$ denotes the first eigenvalue of $-\Delta$ under Dirichlet boundary conditions. In fact by comparing with solutions of $\frac{\partial u}{\partial t}=\Delta u+u(a-u)$, one easily sees that every solution of (1) with nonnegative initial conditions satisfies $u(t) \rightarrow 0$ uniformly on $\Omega$ as $t \rightarrow \infty$. Since we then essentially have a scalar gradient-like equation for $v$, it is easy to obtain a complete picture of the dynamics of (1). (If we add terms $b \cdot \nabla v$, the equation for $v$ may not be gradient-like but, if $\Omega$ is smooth, we can use order arguments to obtain the same result. Similar arguments appear in Conway [5].) Hence interesting behaviour only occurs if $a>\lambda_{1}$. Similarly, we only have interesting behaviour if $d>\lambda_{1}$. We assume that $a>\lambda_{1}$ and $d>\lambda_{1}$ for the remainder of this paper.

If $a>\lambda_{1},(2)$ has a unique nontrivial nonnegative solution $(\bar{u}, 0)$ with second component vanishing identically. Note that $(\bar{u}, 0)$ is independent of $d, e, c$. Similarly, if $d>\lambda_{1}$, there is a unique nontrivial nonnegative solution $(0, \bar{v})$ with first component vanishing identically. Any nonnegative solution $(u, v)$ of $(2)$ other than $(0,0),(\bar{u}, 0)$ or $(0, \bar{v})$ satisfies $u(x)>0$ and $v(x)>$ 0 in $\Omega$. We call such a solution a strictly positive solution. The above results can be found in [8].

Now, as in [8], when $r\left((-\Delta+K I)^{-1}(a+K-c \bar{v}) I\right)$ is less than 1 (or greater than 1), it is independent of $K$ for $K$ such that $a+K-c\|\bar{v}\|_{\infty}>0$. Here $r$ denotes the spectral radius. As in [8], we will abuse notation slightly and write $r\left(-\Delta^{-1}(a-c \bar{v}) I\right)>1 \quad(<1)$ to mean that $r\left((-\Delta+K I)^{-1}(a+K-c \bar{v}) I\right)>1 \quad(<1)$ for all large $K$. For fixed $K>0$, we can argue as in [8, proof of Proposition 1] to deduce that $r\left((-\Delta+K I)^{-1}(a+K-c \bar{v}) I\right)$ is strictly decreasing in $c$ as long as $a+K-c\|\bar{v}\|_{\infty}>0$. (Note that $\bar{v}(x)>0$ in $\Omega$ by the weak maximum principle.) If $c=0$, a simple calculation shows that $r\left(-\Delta^{-1}(a-c \bar{v}) I\right)>1$. We will prove in a moment that $r\left(-\Delta^{-1}(a-c \bar{v})\right)<1$ if $c$ is large. Hence there is a unique positive $\bar{c}$ such that $r\left((-\Delta)^{-1}(a-c \bar{v}) I\right)>1$ if $0 \leq c<\bar{c}$, $r\left((-\Delta)^{-1}(a-c \bar{v}) I\right)=1$ if $c=\bar{c}$, and $r\left((-\Delta)^{-1}(a-c \bar{v}) I\right)<1$ if $c>\bar{c} . \bar{c}$ is a 
function of $a$ and $d$. It remains to prove that $r\left((-\Delta)^{-1}(a-c \bar{v}) I\right)<1$ if $c$ is large. We use positive operator theory as in [8, proof of Proposition 1]. Since $(a-c \bar{v}) \leq(a-c \bar{v})^{+}$

$$
r\left((-\Delta+K I)^{-1}(a+K-c \bar{v}) I\right) \leq r\left((-\Delta+K I)^{-1}\left((a-c \bar{v})^{+}+K\right) I\right) .
$$

Thus it suffices to prove that $r\left((-\Delta+K I)^{-1}\left((a-c \bar{v})^{+}+K\right) I\right)<1$ if $c$ is large. Since $(a-c \bar{v})^{+} \geq 0$, a similar argument to the one presented earlier shows whether $r\left((-\Delta+K I)^{-1}\left((a-c v)^{+}+K\right) I\right)<1$ is independent of $K$ for $K>0$. Thus it suffices to assume that $K=1$. Now $(a-c \bar{v})^{+} \rightarrow 0$ in $L^{p}(\Omega)$ as $c \rightarrow \infty$ for $1<p<\infty$. (Remember that $\bar{v}(x)>0$ in $\Omega$.) Thus, as in the proof of Proposition 1 in [8],

$$
r\left((-\Delta+I)^{-1}\left((a-c \bar{v})^{+}+1\right) I\right) \rightarrow r\left((-\Delta+I)^{-1} I\right)
$$

as $c \rightarrow \infty$. Since a simple calculation shows that $r\left((-\Delta+I)^{-1}\right)<1$, our claim follows.

By similar arguments, there is an $\bar{e}>0$ such that $r\left((-\Delta)^{-1}(d-e \bar{u}) I\right)>1$ if $0 \leq e<\bar{e},=1$ if $e=\bar{e}$, and $<1$ if $e>\bar{e}$. As before $\bar{e}$ is a function of $a$ and $d$.

It can be proved (cf. [8]) that $(\bar{u}, 0)$ is stable (unstable) as a solution of (1) if $r\left((-\Delta+K I)^{-1}(d+K-e \bar{u}) I\right)>1 \quad(<1)$. In other words $(\bar{u}, 0)$ is stable if $e>\bar{e}$ and is unstable if $e<\bar{e}$. A similar result holds for $(0, \bar{v})$ (with $e$ replaced by $c$ ).

Now, if $(c, e) \in[0, \bar{c}) \times[0, \bar{e})$,

$$
r\left((-\Delta)^{-1}(a-c \bar{v}) I\right)>1 \text { and } r\left((-\Delta)^{-1}(d-e \bar{u}) I\right)>1 .
$$

In this case, it is proved in [9] that (2) has a strictly positive solution. In fact, as we see later, there is an asymptotically stable strictly positive solution. If $(c, e) \in(\bar{c}, \infty) \times(\bar{e}, \infty), r\left((-\Delta)^{-1}(a-c \bar{v}) I\right)<1$ and $r\left((-\Delta)^{-1}(d-e \bar{u}) I\right)<1$. Again by [9] it follows that (2) has a strictly positive solution. There need not be a stable strictly positive solution in this case. Thus the question of interest is whether (2) has strictly positive solution for $(c, e)$ in $([\bar{c}, \infty) \times[0, \bar{e}]) \cup((0, \bar{c}] \times$ $[\bar{e}, \infty))$. Note that we are thinking here of the section of the parameter spaces where $a$ and $d$ are fixed.

The main result of this section is the following.

Theorem 1. Assume that $T^{+}=\{(c, e): c>\bar{c}, 0 \leq e<\bar{e}$ and (2) has a strictly positive solution $\}$ is nonempty. Then there exist $\mu>\bar{c}, \nu \in(0, \bar{e})$, and a continuous strictly increasing function $f^{+}:[\bar{c}, \mu] \rightarrow(0, \bar{e}]$ such that $f^{+}(\bar{c})=\nu, f^{+}(\mu)=\bar{e}$, and $T^{+}=\left\{(e, c): c>\bar{c}, 0<e<\bar{e}, e \geq f^{+}(c)\right\}$. Moreover, if $(c, e) \in \operatorname{int} T^{+}$, then (2) has at least two solutions, at least one of which is "asymptotically stable." Moreover, (2) has a strictly positive solution (in fact, an "asymptotically stable" one) if $c=\bar{c}$ and $\nu<e<\bar{e}$, and a strictly positive solution if $e=\bar{e}$ and $\bar{c}<c<\mu$. 
Remarks. 1. By an "asymptotically stable solution" we mean a solution $(u, v)$ such that $r\left(A^{\prime}(u, v)\right) \leq 1,(u, v)$ is an isolated solution, and $(u, v)$ has index 1 in

$D=\left\{(u, v): C_{0}(\bar{\Omega}) \oplus C_{0}(\bar{\Omega}): u \geq 0\right.$ on $\Omega, v \geq 0$ on $\left.\Omega,\|u\|_{\infty}<a,\|v\|_{\infty}<d\right\}$.

Here $A$ is a natural map whose fixed points are the solutions of (2). We will define $A$ a little later. (This is equivalent to the definition of quasi-minimum in [10] but the present notation is more natural here. The equivalence is easy to see if one recalls that the Krein-Rutman theorem (cf. [29, p. 265]) ensures that $r\left(A^{\prime}(u, v)\right) \in \sigma\left(A^{\prime}(u, v)\right)$ provided that $r\left(A^{\prime}(u, v)\right)>0$.) By applying Remark 4 on p. 58 of [10] with $E=L^{p}(\Omega) \oplus L^{p}(\Omega)$ for $p$ large, we deduce that an "asymptotically stable" solution in our sense is indeed an asymptotically stable solution of (1) in the space $X^{\alpha} \oplus X^{\alpha}$ where $X^{\alpha}$ is a fractional power space in the sense of Henry [20, p. 29]. There are two points to be noted here. First, our proof in [10] really shows asymptotic stability. Second, we need to generalize the proof in [10] slightly because $h$ is only $C^{1}$ as a map of $X^{\alpha} \oplus X^{\alpha}$ into $E$ (and not from $E$ into $E$ ). ( $h$ is defined in [10].) However the proof is essentially the same. This result explains our choice of terminology. If $\Omega$ has smooth boundary, it is not difficult to modify the argument in $\S 5$ of Dancer and Hess [16] to obtain asymptotic stability of $(u, v)$ in a direct sum of $L^{p}$ spaces for large $p$.

2. There is an analogous theorem for $T^{-} \equiv\{(c, e): 0 \leq c<\bar{c}, e>\bar{e}$ and (2) has a strictly positive solution $\}$. The curved boundary is of the form $c=f^{-}(e)$. Thus Theorem 1 and our earlier comments provide a good understanding of when (2) has a strictly positive solution. Our ideas in $\S 3$ can be used to show that $f^{+}$need not be convex. It can be proved that there is no strictly positive solution at $(\bar{c}, \tau)$ if $\tau<\nu$ or at $(\gamma, \bar{e})$ if $\gamma>\mu$, and that $T^{+}$is nonempty if (2) has a strictly positive solution for some point $(\bar{c}, \tau)$ where $\tau<\bar{e}$ or $(\gamma, \bar{e})$ with $\gamma>\bar{c}$. We discuss these briefly and some additional results in $\S 2$.

3. It can be shown that $f^{+}, \mu$, and $\nu$ depend continuously on $a$ and $d$. Indeed our methods can be used to obtain results on how $f^{+}$changes with $a$ and $d$.

4. If $a=d$, the results in [6] easily imply that $T^{+}$and $T^{-}$are both empty and the only points of $(\{\bar{c}\} \times R) \cup(R \times\{\bar{e}\})$ where there is a strictly positive solution is $(\bar{c}, \bar{e})$. On the other hand, we prove in $\S 2$ that for most $(a, d)$ either $T^{+}$or $T^{-}$is nonempty.

5. Some explicit estimates for the size of $T^{+}$follow from [24]. Theorem 1 answers a question in [24].

We will prove Theorem 1 by a series of lemmas. If we use the cone $\widetilde{K}=$ $\left\{(u, v) \in C_{0}(\bar{\Omega}) \times C_{0}(\bar{\Omega}): u \geq 0\right.$ in $\Omega, v \leq 0$ in $\left.\Omega\right\}$ and the corresponding order (denoted by $\geq_{S}$ ), we easily see that, for $K$ large, the map $A$ defined by

$$
A(u, v)=(-\Delta+K I)^{-1}(u(a+K-u-c v), v(d+K-v-e u))
$$


is increasing on

$$
\begin{array}{r}
D=\left\{(u, v) \in C_{0}(\bar{\Omega}) \times C_{0}(\bar{\Omega}):\|u\|_{\infty}<a,\|v\|_{\infty}<a, \quad u \geq 0 \text { on } \Omega,\right. \\
v \geq 0 \text { on } \Omega\}
\end{array}
$$

Here we have abused notation slightly by identifying the scalar operator $(-\Delta+K I)^{-1}$ and the operator which operates on a two-component vector by operating as $(-\Delta+K I)^{-1}$ on each component. The above result is easily shown by noting that $A$ is Fréchet differentiable and $A^{\prime}(u, v)$ maps $\widetilde{K}$ into itself for each $(u, v) \in K$. Indeed, one can easily prove that $A^{\prime}(u, v)$ is a demi-interior operator in the sense of $[10$, p. 50] when $u>0$ in $\Omega$ and $v>0$ in $\Omega$. Here we use Lemma 2 in [8] and that $(u, v) \in \widetilde{K}$ is demi-interior to $\widetilde{K}$ if and only if $u$ and $-v$ are both demi-interior to the usual cone $K_{1}$ in $C_{0}(\bar{\Omega})$. These remarks enable us to use a number of standard results in cone theory.

Lemma 1. Assume that $\left(c_{1}, e_{1}\right) \in T^{+}$. If $0 \leq c \leq c_{1}, e \geq e_{1}, e<\bar{e}$, and either $c<c_{1}$ or $e>e_{1}$ then (2) has a strictly positive solution which is an "asymptotically stable" solution of (1). Moreover, if $c>\bar{c}$, there must be at least one other strictly positive solution.

Proof. We first prove the existence of a strictly positive solution. Since $\left(c_{1}, e_{1}\right)$ $\in T^{+}$, there exists a strictly positive solution $\left(u_{1}, v_{1}\right)$ of

$$
-\Delta u=u\left(a-u-c_{1} v\right), \quad-\Delta v=v\left(d-v-e_{1} u\right) .
$$

By standard estimates (cf. [8, §2]), $u_{1} \leq \bar{u}$ and $v \leq \bar{v}$. Thus $\left(u_{1}, v_{1}\right) \in D$ and $\left(u_{1}, v_{1}\right) \leq_{S}(\bar{u}, 0)$ (for the order generated by $\left.\widetilde{K}\right)$. Now

$$
-\Delta u_{1}=u_{1}\left(a-u_{1}-c_{1} v_{1}\right) \leq u_{1}\left(a-u_{1}-c v_{1}\right)
$$

since $c \leq c_{1}$. Moreover, strict inequality holds on $\Omega$ if $c<c_{1}$ since $u_{1}>0$ on $\Omega$ and $v_{1}>0$ on $\Omega$. Thus

$$
u_{1} \leq(-\Delta+K I)^{-1}\left(u_{1}\left(a+K-u_{1}-c v_{1}\right)\right)
$$

and equality does not hold if $c<c_{1}$. Similarly,

$$
v_{1} \geq(-\Delta+K I)^{-1}\left(v_{1}\left(d+K-v_{1}-e u_{1}\right)\right)
$$

and equality does not hold if $e>e_{1}$. Hence $A\left(u_{1}, v_{1}\right)>_{s}\left(u_{1}, v_{1}\right)$ (where $>_{S}$ means $\geq_{S}$ and equality does not hold). Hence we have that $\left(u_{1}, v_{1}\right) \leq_{S}$ $(\bar{u}, 0), A\left(u_{1}, v_{1}\right)>_{S}\left(u_{1}, v_{1}\right), A(\bar{u}, 0)=(\bar{u}, 0)$ (since $(\bar{u}, 0)$ is a solution of (2)), and $A$ is increasing on the order interval $C=\left[\left(u_{1}, v_{1}\right),(\bar{u}, 0)\right]$. Hence the iterates $\left(u_{n+1}, v_{n+1}\right)=A\left(u_{n}, v_{n}\right)$ will increase (in $\left.\tilde{K}\right)$ to a fixed point $(\tilde{u}, \tilde{v})$ of $A$ (and thus a solution of (2)) with $u_{1} \leq \tilde{u} \leq \bar{u}$ and $0 \leq \tilde{v} \leq \bar{v}$. This will be a strictly positive solution of (2) unless $\tilde{v}=0$. In this case, the first equation of (2) implies that $\tilde{u}=\bar{u}$. (Note that $\tilde{u}$ is nontrivial since $\tilde{u} \geq u_{1}$.)

Since the iterates $\left\{\left(u_{n}, v_{n}\right)\right\}$ converge to the minimal fixed point of $A$ in $C$, we see that in this case $(\bar{u}, 0)$ will be the only fixed point in $C$. Now $C$ is closed and convex (and thus contractible) and $A C \subseteq C$ by the monotonicity. 
Hence by basic properties of the fixed point index, the sum of the indices of the fixed points of $A$ in $C$ (counted relative to $C$ ) is 1 . Since $(\bar{u}, 0)$ is the only fixed point in $C$, it follows that index ${ }_{C}(A,(\bar{u}, 0))=1$. However, since $u_{1}(x)<\bar{u}(x)$ and $v_{1}(x)>0$ in $\Omega$, it is easy to see that $\bar{C}_{(\bar{u}, 0)}=-\widetilde{K}$ where our notation follows $\S \S 1$ and 2 of [11]. Thus, by Theorem 1 and Lemma 2 in [11] and Proposition 1 in [10], $\operatorname{index}_{C}(A,(\bar{u}, 0))=0$ if we prove that $r\left(A^{\prime}(\bar{u}, 0)\right)>1$ and $A^{\prime}(\bar{u}, 0)$ does not have an eigenvector in $\widetilde{K}$ corresponding to the eigenvalue 0 . Hence, if we prove these properties of $A^{\prime}(\bar{u}, 0)$, we will have a contradiction and hence there will be a strictly positive solution. This is very similar to part of the proof of Theorem 1(ii) in [8]. Now, by a simple calculation,

$$
A^{\prime}(\bar{u}, 0)(h, k)=(-\Delta+K I)^{-1}((a+K-2 \bar{u}) h-c \bar{u} k,(d+K-e \bar{u}) k) .
$$

It follows easily from this formula that

$$
\begin{aligned}
\sigma\left(A^{\prime}(\bar{u}, 0)\right)= & \sigma\left((-\Delta+K I)^{-1}(a+K-2 \bar{u}) I\right) \\
& \cup \sigma\left((-\Delta+K I)^{-1}(d+K-e \bar{u}) I\right) .
\end{aligned}
$$

Hence

$$
r\left(A^{\prime}(\bar{u}, 0)\right) \geq r\left((-\Delta+K I)^{-1}(d+K-e \bar{u}) I\right)>1 \text { since } e<\bar{e} .
$$

Hence $r\left(A^{\prime}(\bar{u}, 0)\right)>1$. That $A^{\prime}(\bar{u}, 0)(h, k) \neq(h, k)$ if $(h, k) \in \widetilde{K} \backslash\{0,0\}$ is almost the same as the proof of the corresponding result at the bottom of $\mathrm{p}$. 733 of [8] once we recall that $r\left((-\Delta+K I)^{-1}(d+K-e \bar{u}) I\right)>1$.

This completes the proof of the existence of the strictly positive solution $(u, v)$.

Before starting the remainder of the proof, we note that an isolated strictly positive solution has the same index in $D$ or $E=C_{0}(\bar{\Omega}) \oplus C_{0}(\bar{\Omega})$ or in $K_{2}=$ $K_{1} \oplus K_{1}$ or in $C$ if it belongs to $C$. We will return and prove this at the end of the proof.

Now, to prove the existence of a second strictly positive solution if $c>\bar{c}$, we note that there is nothing to prove unless there is a unique strictly positive fixed point $(\tilde{u}, \tilde{v})$ of $A$ in $C$. Since, as before, the sum of the indices of the fixed points in $C$ is 1 and since, as before, $(\bar{u}, 0)$ has index 0 in $C$, it follows that $\operatorname{index}_{C}(A,(\tilde{u}, \tilde{v}))=1$. Hence, by the previous paragraph, $\operatorname{index}_{K_{2}}(A,(\tilde{u}, \tilde{v}))=1$. However, if $e<\bar{e}$ and $c>\bar{c}, r\left((-\Delta)^{-1}(a-c \bar{v}) I\right)<1$ and $r\left((-\Delta)^{-1}(d-e \bar{u}) I\right)>1$. Thus, by the arguments in $\S 2$ of [8], the sum of the indices of the strictly positive solutions is 0 (relative to $K_{2}$ ). Hence there must be another strictly positive solution, as required.

We now prove that there is an "asymptotically stable" solution in $C$. We have that $A\left(u_{1}, v_{1}\right)>_{S}\left(u_{1}, v_{1}\right)$ and $A(\bar{u}, 0)=(\bar{u}, 0)$. Let

$$
w=A\left(u_{1}, v_{1}\right)-\left(u_{1}, v_{1}\right)>_{S} 0 .
$$


Since $A$ is increasing, we see that if $0<t<1$, the map $A_{t}$ defined by $A_{t}(u, v)=A(u, v)-t w$ is an increasing $C^{1}$ map of $C$ into itself. Let $x_{t}$ denote its minimal fixed point in $C$. This is obtained by the obvious iteration from $\left(u_{1}, v_{1}\right)$. Moreover, by the iteration, $x_{t}$ increases as $t$ decreases. Since $\left\{x_{t}: t \in(0,1)\right\}$ lies in a compact set (by the boundedness of $C$ and the compactness of $A$ ), we see easily that $x_{0}=\lim _{t \rightarrow 0^{+}} x_{t}$ exists, is in $C$, and is a fixed point of $A$. We will prove that $x_{0}$ is "asymptotically stable". Since $x_{t} \geq_{S}\left(u_{1}, v_{1}\right)$, the first component of $x_{t}$ is positive in $\Omega$. Since $x_{t}=A x_{t}-$ $t w \leq_{S} A(\bar{u}, 0)-t w=(\bar{u}, 0)-t w$ and since both components of $w$ are positive on $\Omega$, it follows that the second component of $x_{t}$ is positive on $\Omega$. Hence, by our earlier comments, $A^{\prime}\left(x_{t}\right)$ is a demi-interior operator. Hence $r\left(A^{\prime}\left(x_{t}\right)\right) \leq 1$. This follows by applying the remarks at the bottom of $\mathrm{p} .143$ in [11] to $A_{t}$. The only point to note is that since $A^{\prime}\left(x_{t}\right)$ is a demi-interior operator, it follows easily that any eigenvector $f$ of $A^{\prime}\left(x_{t}\right)^{*}$ in the dual cone $\widetilde{K}^{*}$ is strictly positive, that is $f(x)>0$ if $x \in \widetilde{K} \backslash\{0\}$. (See, for example, [10, p. 50].) Since $r\left(A^{\prime}\left(x_{t}\right)\right) \leq 1$, we see from the continuity of the spectral radius that $r\left(A^{\prime}\left(x_{0}\right)\right) \leq 1$. Thus, since $r\left(A^{\prime}(\bar{u}, 0)\right)>1, x_{0} \neq(\bar{u}, 0)$. Hence $x_{0}$ is a strictly positive solution. Suppose that $x_{0}$ is isolated in $C$. (We will prove this in a moment.) By our earlier comments, $A^{\prime}\left(x_{0}\right)$ is a demi-interior operator and $r\left(A^{\prime}\left(x_{0}\right)\right) \leq 1$. We can now prove that index ${ }_{C}\left(A, x_{0}\right)=1$ by a similar argument to the proof of a corresponding result in Proposition 3 and Remark 4 in [11]. As there, the result is easy unless $r\left(A^{\prime}\left(x_{0}\right)\right)=1$. In that case, as in [11], we easily see that the solutions $(x, t)$ of $x=A(x)-t w$ near $\left(x_{0}, 0\right)$ are of the form $\left(x_{0}+\alpha h+w(\alpha), \phi(\alpha)\right)$ where $w$ and $\phi$ are $C^{1}, w(0)=0, \phi(0)=0, h$ spans $N\left(I-A^{\prime}\left(x_{0}\right)\right), f$ spans $N\left(I-A^{\prime}\left(x_{0}\right)^{*}\right)$, and $f(w(\alpha))=0$ for all small $\alpha$. The proof is the same as the one there except for two points. First, because $A_{t}$ only maps $C$ into itself for $t \geq 0$, we only know that $\alpha h+w(\alpha) \in C$ for $\alpha \leq 0$. Note that, since $x_{t}<_{S} x_{0}, x_{t}$ corresponds to a negative $\alpha$. Second, as a consequence, if $\alpha>0$, we only find that either $\phi(\alpha)<0$ or $\alpha h+w(\alpha) \notin C$. (We do find that $\phi(\alpha)>0$ for $\alpha<0$.) The only other point is that the sign in a number of other inequalities is reversed. Hence we find that $\operatorname{index}_{C}\left(A, x_{0}\right)=1$ and thus, by a comment earlier in the proof, index ${ }_{D}\left(A, x_{0}\right)=1$.

Hence $x_{0}$ will be "asymptotically stable" if it is isolated. Now, as in the previous paragraph, the solutions of $x=A(x)-t w$ near $\left(x_{0}, 0\right)$ in $E \times R$ are $\left\{\left(x_{0}+\alpha h+w(\alpha), \phi(\alpha)\right):|\alpha| \leq \varepsilon\right\}$. By the argument of Remark 5 on $\mathrm{p}$. 143 of [11], if $x_{0}$ is not isolated, $\phi(\alpha)=0$ for all small $\alpha$. (Note that $A$ is a continuous polynomial map on $E$ and hence is real analytic.) Thus any solution of $x=A(x)-t w$ near $\left(x_{0}, 0\right)$ has $t=0$. This is impossible since $\left(x_{t}, t\right)$ is such a solution. Thus $x_{0}$ is an isolated fixed point of $A$ in $E$.

It remains to prove our claim that the indices of fixed points is the same in the different spaces. It is here that we use $\partial \Omega$ is smooth. We use the space $V \otimes V$ where $V$ denotes the space of functions $u$ in $C_{0}(\bar{\Omega})$ for which $\phi_{1}^{-1} u$ extends to 
a continuous function on $\bar{\Omega}$ with the norm $\|u\|^{\prime}=\sup _{x \in \Omega}\left|\phi_{1}^{-1}(x) u(x)\right|$, where $\phi_{1}$ denotes the positive eigenfunction corresponding to the first eigenvalue of $-\Delta$ on $\Omega$ under Dirichlet boundary conditions. It is easy to check (cf. [1]) that $V$ is a Banach space under the norm \|\|$^{\prime}$. Let $K_{4}$ denote the cone $K_{2} \cap V$. It is easy to see that $K_{4}$ has nonempty interior in $V$ and that the interior elements are functions $u$ for which $\inf _{x \in \Omega} \phi_{1}^{-1} u(x)>0$. In particular, this holds if $u$ is $C^{1}$ on $\bar{\Omega}, u(x)>0$, and $\frac{\partial u}{\partial n}<0$ on $\partial \Omega$. Now, by a similar argument to that in [1], one easily sees that $A$ is a completely continuous map of $E$ into $V \oplus V$. Moreover, if $x$ is an isolated fixed point of $A$ in $E$, the commutativity theorem for the degree (cf. [19 or 27]) ensures that index $\operatorname{den}_{E}(A, x)=\operatorname{index}_{V \oplus V}(A, x)$. Similarly, if $x \in C$, the index of $x$ in $C$ is the same as that in $C \cap(V \oplus V)$. This means that we need only prove our results on indices on the space $V \oplus V$. In this case, we simply have to prove that the point is interior to $C \cap(V \oplus V)$ or $D \cap(V \oplus V)$ and the result is then obvious. This is easy to check once we note that, if $u_{2}>u_{1}$ and $v_{2}<v_{1}$ in $K_{2}$, then $A\left(u_{2}, v_{2}\right)-A\left(u_{1}, v_{1}\right)$ is interior to $(V \oplus V) \cap \widetilde{K}$ (because the maximum principle ensures that each component has nonzero normal derivative on $\partial \Omega$ ). Note that our earlier ideas show easily that each component of $A\left(u_{2}, v_{2}\right)-A\left(u_{1}, v_{1}\right)$ is nonzero on $\Omega$. This completes the proof of Lemma 1.

Remarks. 1. The existence of a strictly positive solution in the lemma can also be proved by using the iteration scheme in [9, pp. 241-242], though this seems to work less well in degenerate cases. This method has the advantage that it implies that the "asymptotically stable" solution is as symmetric as $\Omega$ and that $f^{+}$is unchanged if we look at solutions with the same symmetries as $\Omega$.

2 . As we noted, the result is true without the smoothness of $\partial \Omega$. To prove this, we need to use some of the ideas in $[11,10]$. However, it is a little tedious because we cannot apply the results there directly but have to modify the techniques very slightly. Part of the difficulty is that $A^{\prime}(u, v)$ is not always a demi-interior operator.

3. We essentially used the analyticity to prove the existence of the asymptotically stable solution. If we have a nonlinearity which is similarly behaved to ours but is not real analytic, one can prove instead the existence of a stable solution and a set of solutions whose index is nonzero. (This would suffice for our other arguments.) A slight modification of our analyticity arguments imply that, if either $e \neq \bar{e}$ or $c \neq \bar{c}$, then every strictly positive solution $(u, v)$ with $r\left(A^{\prime}(u, v)\right) \leq 1$ is isolated.

4. It is possible to give another proof of Lemma 1 when $\Omega$ is smooth by combining some of the ideas in Dancer and Hess [16] with the ideas in [11] to construct an asymptotically stable solution and then use a variant of the ideas of Matano [26] to find another solution between the asymptotically stable solution and $(0, \bar{v})$.

Lemma 2. There exists $\varepsilon>0$ such that there is no strictly positive solution of (2) if $c \geq \bar{c}, e \leq \bar{e}$, and either $e \leq \varepsilon$ or $c \geq \varepsilon^{-1}$. 
Proof. We first prove that there is no strictly positive solution if $e$ is small. Suppose that $e_{n} \leq n^{-1}$ and (2) has a strictly positive solution $\left(u_{n}, v_{n}\right)$ for $c=c_{n}$ and $e=e_{n}$ (where $c_{n} \geq \bar{c}$ ). By the obvious bounds, we see that $\left\|u_{n}\right\|_{\infty} \leq a$ and $\left\|v_{n}\right\|_{\infty} \leq d$. By the compactness of $(-\Delta)^{-1}$ on $C_{0}(\bar{\Omega})$, we see from the second equation that $\left\{v_{n}\right\}$ is compact in $C_{0}(\bar{\Omega})$. Thus, by choosing a subsequence if necessary, we can assume that $v_{n} \rightarrow v$ in $C_{0}(\bar{\Omega})$ as $n \rightarrow \infty$. Since $e_{n} u_{n} \rightarrow 0$ as $n \rightarrow \infty$, we can pass to the limit in the second equation and deduce that $v$ is a nonnegative solution of

$$
-\Delta v=v(d-v) \quad \text { in } \Omega, \quad v=0 \quad \text { on } \partial \Omega .
$$

Thus $v=0$ or $v=\bar{v}$. In the first case, this implies that $v_{n} \rightarrow 0$ in $C_{0}(\bar{\Omega})$ as $n \rightarrow \infty$. Now, by the second equation,

$$
v_{n}=(-\Delta+K I)^{-1}\left(\left(d+K-v_{n}-e_{n} u_{n}\right) v_{n}\right) .
$$

Since $(-\Delta+K I)^{-1}(a(x) I)$ is a demi-interior operator on $C_{0}(\bar{\Omega})$ if $a(x)>0$ on $\bar{\Omega}$ and thus has its spectral radius as the only nonzero point of the spectrum to which there corresponds a positive eigenfunction,

$$
r\left((-\Delta+K I)^{-1}\left(d+K-v_{n}-e_{n} u_{n}\right) I\right)=1 \quad(\text { where } K \geq 0) .
$$

Passing to the limit as $n \rightarrow \infty$ and by using the continuity of the spectral radius, we see that $r\left((-\Delta+K I)^{-1}(d+K) I\right)=1$. An easy calculation shows that this is false. Hence $v_{n} \rightarrow \bar{v}$ as $n \rightarrow \infty$.

Now, by similar arguments to above applied to the first equation,

$$
r\left((-\Delta+K I)^{-1}\left(a+K-u_{n}-c_{n} v_{n}\right) I\right)=1 .
$$

Now, $a-u_{n}-c_{n} v_{n} \leq\left(a-c_{n} v_{n}\right)^{+}$. Hence, by spectral properties of positive linear operators,

$$
\begin{aligned}
1 & =r\left((-\Delta+K I)^{-1}\left(a+K-u_{n}-c_{n} v_{n}\right) I\right) \\
& \leq r\left((\Delta+K I)^{-1}\left(K+\left(a-c_{n} v_{n}\right)^{+}\right) I\right) .
\end{aligned}
$$

In particular, if $c_{n} \rightarrow \infty$ as $n \rightarrow \infty$, then, since $v_{n} \rightarrow \bar{v}$ as $n \rightarrow \infty$ and $\bar{v}(x)>0$ in $\Omega$, we easily see that $\left(a-c_{n} v_{n}\right)^{+} \rightarrow 0$ in $L^{p}(\Omega)$ as $n \rightarrow \infty$ for every $p$ in $(1, \infty)$. Thus (cf. [8, p. 735]), $r\left((-\Delta+K I)^{-1}\left(K+\left(a-c_{n} v_{n}\right)^{+}\right) I\right) \rightarrow$ $r\left((-\Delta+K I)^{-1} K\right)<1$ as $n \rightarrow \infty$. Hence we have a contradiction unless $\left\{c_{n}\right\}$ is bounded. Thus, by choosing a subsequence, if necessary, we can ensure that $c_{n} \rightarrow \hat{c} \geq \bar{c}$ as $n \rightarrow \infty$. Now, by passing to the limit in the first equation much as earlier in the proof, we deduce that, by choosing a subsequence if necessary, $u_{n} \rightarrow \tilde{u}$ in $C_{0}(\bar{\Omega})$ as $n \rightarrow \infty$ where $-\Delta \tilde{u}=\tilde{u}(a-\tilde{u}-\hat{c} \bar{v})$ in $\Omega$. Now it is easy to see (cf. [8, Lemma 1] for a similar result), this equation can only have a nontrivial nonnegative solution (with $u=0$ on $\partial \Omega$ ) if $r\left((-\Delta)^{-1}(a-\hat{c} \bar{v}) I\right)>1$. However, since $\hat{c} \geq \bar{c}, r\left((-\Delta)^{-1}(a-\hat{c} \bar{v}) I\right) \leq 1$ with strict inequality if $\hat{c}>\bar{c}$. Hence $\tilde{u}=0$ and thus $u_{n} \rightarrow 0$ as $n \rightarrow \infty$. Since

$$
r\left((-\Delta+K I)^{-1}\left(a+K-u_{n}-c_{n} v_{n}\right) I\right)=1,
$$


we see by passing to the limit that $r\left((-\Delta+K I)^{-1}(a+K-\hat{c} \bar{v}) I\right)=1$. By the definition of $\bar{c}$, this implies that $\hat{c}=\bar{c}$. Hence we have that $u_{n} \rightarrow 0, v_{n} \rightarrow \bar{v}$, $e_{n} \rightarrow 0$, and $c_{n} \rightarrow \bar{c}$ as $n \rightarrow \infty$ and $c_{n} \geq \bar{c}$. Thus we are looking at strictly positive solutions bifurcating from $(0, \bar{v})$. That this is impossible (for $c_{n} \geq \bar{c}$ ) follows from a simple bifurcation analysis very similar to the one at the start of $\S 2$ below (or $\S 3$ of [4]). This proves our claim for $e$ small.

We now consider the case where $c$ is large. Thus we assume that there exist strictly positive solutions $\left(u_{n}, v_{n}\right)$ for $c=c_{n}$ and $e=e_{n}$ where $0 \leq e_{n} \leq \bar{e}$ and $c_{n} \rightarrow \infty$ as $n \rightarrow \infty$. By the previous part, $e_{n}$ is not small. Thus, by choosing a subsequence if necessary we can ensure that $e_{n} \rightarrow \hat{e}$ as $n \rightarrow \infty$ where $0 \leq \hat{e} \leq \bar{e}$. By working with the second equation much as before, we can assume that $v_{n} \rightarrow \tilde{v}$ in $C_{0}(\bar{\Omega})$. Moreover, since $\left\|u_{n}\right\|_{\infty} \leq a$ for all $n$, we can assume that $u_{n}$ tends (weakly) to $u_{0}$ in $L^{p}(\Omega)$ for $1<p<\infty$ where $u_{0} \in L^{\infty}(\Omega)$. By passing to the limit in the second equation, we see that $\tilde{v}$ is a nonnegative solution of $-\Delta v=v\left(d-v-\hat{e} u_{0}\right)$ in $\Omega$. By the weak Harnack inequality (cf. [18, Corollary 8.21]), $\tilde{v}(x)>0$ in $\Omega$ or $\tilde{v} \equiv 0$. We now show that the first case is impossible. This is similar to earlier. By the first equation,

$$
\begin{aligned}
1 & =r\left((-\Delta+K I)^{-1}\left(a+K-u_{n}-c_{n} v_{n}\right) I\right) \\
& \leq r\left((-\Delta+K I)^{-1}\left(K+\left(a-c_{n} v_{n}\right)^{+}\right) I\right)
\end{aligned}
$$

(since $\left.a+K-u_{n}-c_{n} v_{n} \leq K+\left(a-c_{n} v_{n}\right)^{+}\right)$.

However the right-hand side tends to $r\left((-\Delta+K I)^{-1} K I\right)<1$ as $n \rightarrow \infty$. (This follows because $\left(a-c_{n} v_{n}\right)^{+} \rightarrow 0$ in $L^{p}(\Omega)$ for all $p$ as $n \rightarrow \infty$ since $c_{n} \rightarrow \infty$ as $n \rightarrow \infty$ and $v_{n}(x) \rightarrow \tilde{v}(x)>0$ as $n \rightarrow \infty$.) Hence we have a contradiction and thus $\tilde{v} \equiv 0$, that is, $v_{n} \rightarrow 0$ as $n \rightarrow \infty$. By the second equation

$$
\begin{aligned}
1 & =r\left((-\Delta+K I)^{-1}\left(d+K-v_{n}-e_{n} u_{n}\right) I\right) \\
& \geq r\left((-\Delta+K I)^{-1}(d+K-\varepsilon-(\hat{e}+\varepsilon) \bar{u}) I\right) \quad \text { if } n \text { is large }
\end{aligned}
$$

(since $u_{n} \leq \bar{u}, e_{n} \rightarrow \hat{e}$, and $\left\|v_{n}\right\|_{\infty} \rightarrow 0$ as $n \rightarrow \infty$ ).

However, as $\varepsilon \rightarrow 0$,

$$
\begin{aligned}
& r\left((-\Delta+K I)^{-1}(d+K-\varepsilon-(\hat{e}+\varepsilon) \bar{u}) I\right) \\
& \quad \rightarrow r\left((-\Delta+K I)^{-1}(d+K-\hat{e} \bar{u}) I\right)>1 \text { if } \hat{e}<\bar{e} .
\end{aligned}
$$

Equations (6) and (7) give a contradiction if $\hat{e}<\bar{e}$. Thus the only case left to consider is when $e_{n} \rightarrow \bar{e}$ as $n \rightarrow \infty$ and $v_{n} \rightarrow 0$ as $n \rightarrow \infty$. Let $w_{n}=$ $\left(\left\|v_{n}\right\|_{\infty}\right)^{-1} v_{n}$. By the equation for $v_{n}, \Delta w_{n}$ is bounded. Thus $\left\{w_{n}\right\}$ is compact in $C_{0}(\bar{\Omega})$. Thus, by choosing a subsequence if necessary, we can assume that $w_{n} \rightarrow w$ in $C_{0}(\bar{\Omega})$ as $n \rightarrow \infty$. Since $\left\|w_{n}\right\|_{\infty}=1,\|w\|_{\infty}=1$. Thus $w \neq 0$. By passing to the limit in the equation for $w_{n},-\Delta w=w\left(d-\bar{e} u_{0}\right)$ in $\Omega$. Since $w \geq 0$ in $\Omega$ and $w$ does not vanish identically, it follows from the weak Harnack inequality that $w(x)>0$ in $\Omega$. We can now deduce that $\left\{c_{n}\left\|v_{n}\right\|_{\infty}\right\}$ 
is bounded. If $c_{n}\left\|v_{n}\right\|_{\infty} \rightarrow \infty$ as $n \rightarrow \infty$,

$$
\begin{aligned}
1 & =r\left((-\Delta+K I)^{-1}\left(a+K-u_{n}-c_{n}\left\|v_{n}\right\|_{\infty} w_{n}\right) I\right) \\
& \leq r\left((-\Delta+K I)^{-1}\left(K+\left(a-c_{n}\left\|v_{n}\right\|_{\infty} w_{n}\right)^{+}\right) I\right)
\end{aligned}
$$

and, since $c_{n}\left\|v_{n}\right\|_{\infty} \rightarrow \infty$ as $n \rightarrow \infty$ ensures that $\left(a_{n}-c_{n}\left\|v_{n}\right\|_{\infty} w_{n}\right)^{+} \rightarrow 0$ in $L^{p}(\Omega)$, we can obtain a contadiction as before. (Remember that $w_{n}(x) \rightarrow$ $w(x)>0$ as $n \rightarrow \infty$.) By choosing a subsequence, we can ensure that $\left\{c_{n}\left\|v_{n}\right\|_{\infty}\right\}$ converges and thus $\left\{c_{n} v_{n}\right\}$ converges in $C_{0}(\bar{\Omega})$ (since $\left\{w_{n}\right\}$ converges). Let $\tilde{w}_{n}=c_{n} v_{n}$. Then

$$
-\Delta u_{n}=u_{n}\left(a-u_{n}-\tilde{w}_{n}\right), \quad-\Delta \tilde{w}_{n}=\tilde{w}_{n}\left(d-\mu_{n} \tilde{w}_{n}-e_{n} u_{n}\right),
$$

where $\mu_{n}=\left(c_{n}\right)^{-1}$. Note that $\mu_{n} \rightarrow 0$ as $n \rightarrow \infty$. Since $\left\{\tilde{w}_{n}\right\}$ is bounded, we can easily deduce from the first equation that, by choosing a subsequence if necessary, $\left\{u_{n}\right\}$ converges. Hence we can assume that $\left(u_{n}, \tilde{w}_{n}\right) \rightarrow(\hat{u}, \hat{w})$ in $E$ as $n \rightarrow \infty$. By passing to the limit, we deduce that $(\hat{u}, \hat{w})$ is a nonnegative solution of

$$
-\Delta \hat{u}=\hat{u}(a-\hat{u}-\hat{w}), \quad-\Delta \hat{w}=\hat{w}(d-\bar{e} \hat{u})
$$

in $\Omega$ with $\hat{u}=\hat{w}=0$ on $\partial \Omega$. Since the second equation of $(8)$ implies that

$$
r\left((-\Delta+K I)^{-1}\left(d+K-\mu_{n} \tilde{w}_{n}-e_{n} u_{n}\right) I\right)=1,
$$

we see in the limit that

$$
r\left((-\Delta+K I)^{-1}(d+K-\bar{e} \hat{u}) I\right)=1 .
$$

Hence $\hat{u} \neq 0$. By the first equation of $(9), \hat{u} \leq \bar{u}$ and $\hat{u}(x)<\bar{u}(x)$ in $\Omega$ unless $\hat{w}=0$. However if $\hat{u}(x)<\bar{u}(x)$ in $\Omega_{1}$,

$$
r\left((-\Delta+K I)^{-1}(d+K-\bar{e} \hat{u}) I\right)>r\left((-\Delta+K I)^{-1}(d+K-\overline{e u}) I\right)=1
$$

by the definition of $\bar{e}$. Note, to obtain the strict inequality, we have used a similar argument to that in [8, top of p. 735]. Since (11) contradicts (10), we have that $\hat{u}=\bar{u}$ and $\hat{w}=0$. Hence $\left(u_{n}, w_{n}\right) \rightarrow(\bar{u}, 0)$ in $E$ as $n \rightarrow \infty$. It is now a standard bifurcation analysis of $(8)$ near $(\bar{u}, 0)$ to show that this is impossible if $e_{n} \leq \bar{e}$ for all $n$. We sketch the proof (the quickest but not the most standard proof). By the second equation, $h_{n}=\left(\left\|\tilde{w}_{n}\right\|_{\infty}\right)^{-1} \tilde{w}_{n}$ converges to the positive eigenfunction $h$ of

$$
-\Delta h=h(d-\bar{e} \bar{u})
$$

in $\Omega, h=0$ on $\partial \Omega$ (where $\|h\|_{\infty}=1$ ). By the first equation,

$$
-\Delta\left(\bar{u}-u_{n}\right)+q_{n}\left(\bar{u}-u_{n}\right)=\tau_{n} u_{n} h_{n}
$$

where $\tau_{n}=\left\|\tilde{w}_{n}\right\|_{\infty}$ and $q_{n}=\bar{u}+u_{n}-a$. Hence

$$
\bar{u}-u_{n}=\tau_{n} L(\bar{u} h)+o\left(\tau_{n}\right)
$$


where $L$ is the inverse of $-\Delta+(2 \bar{u}-a) I$ (for Dirichlet boundary conditions). Here we are using that $-\Delta+(2 \bar{u}-a) I$ is invertible. This follows by a standard comparison argument (cf. $[4, \S 3]$ or [8]). Moreover $L$ is a positive operator (cf. [4]). Now, by taking the scalar product of the second equation of (8) with $\tau_{n}^{-1} h$, we see that

$$
-\mu_{n} \tau_{n}\left\langle h_{n}^{2}, h\right\rangle-\left(e_{n}-e\right)\left\langle u_{n} h_{n}, h\right\rangle-\bar{e}\left\langle\left(u_{n}-\bar{u}\right) h_{n}, h\right\rangle=0 .
$$

Here, $\langle$,$\rangle is the usual scalar product on L^{2}(\Omega)$. Hence, by our formula for $\bar{u}-u_{n}$,

$$
\left(e_{n}-e\right)\left\langle u_{n} h_{n}, h\right\rangle=\bar{e} \tau_{n}\left\langle L(\bar{u} h) h_{n}, h\right\rangle+o\left(\tau_{n}\right) .
$$

Recall that $\mu_{n} \rightarrow 0$ as $n \rightarrow \infty$. Now

$$
\left\langle L(\bar{u} h) h_{n}, h\right\rangle \rightarrow\left\langle L(\bar{u} h), h^{2}\right\rangle>0 \text { as } n \rightarrow \infty
$$

since $L$ is a positive operator. Hence, since $\tau_{n}>0$, the right-hand side of (12) has the same sign as $\left\langle L(\bar{u} h) h_{n}, h\right\rangle$ and thus is positive. Since $\left\langle u_{n} h_{n}, h\right\rangle>0$ (because $h(x)>0$ on $\Omega$ ), it follows that $e_{n}-e>0$ for large $n$. This is impossible because we are assuming that $e_{n} \leq e$. Hence we have a contradiction and our claim is proven.

Remarks. Unlike most of our proofs, this does use a little on the structure of our nonlinearity. However, it can be easily modified to apply to a wide case of nonlinearities including Rozenweig-Macarthur type nonlinearities. (For the second part, we may need to scale $v$ differently.)

Lemma 3. $T^{+}$is closed in $(\bar{c}, \infty) \times(0, \bar{e})$.

Proof. Assume that $\left(c_{n}, e_{n}\right) \in T^{+}$and $\left(c_{n}, e_{n}\right) \rightarrow(\tilde{c}, \tilde{e}) \in(\bar{c}, \infty) \times(0, \bar{e})$ as $n \rightarrow \infty$. Let $\left(u_{n}, v_{n}\right)$ be a strictly positive solution for $c=c_{n}$ and $e=$ $e_{n}$. Since $\left\|u_{n}\right\|_{\infty} \leq a$ and $\left\|v_{n}\right\|_{\infty} \leq d$, a standard argument ensures that a subsequence of $\left(u_{n}, v_{n}\right)$ converges to a nonnegative solution $(\tilde{u}, \tilde{v})$ of (2). Then $(\tilde{u}, \tilde{v})$ is $(0,0)$ or $(0, \bar{v})$ or $(\bar{u}, 0)$ or a strictly positive solution of $(2)$ for $c=\tilde{c}$ and $e=\tilde{e}$. If we exclude the first three possibilities, $(\tilde{c}, \tilde{e}) \in T^{+}$ and the proof is completed. We exclude the second. The others are similar. By the equation for $u_{n}, r\left((-\Delta+K)^{-1}\left(a+K-u_{n}-e_{n} v_{n}\right) I\right)=1$. Hence, by letting $n$ tend to infinity, $r\left((-\Delta+K I)^{-1}(a+K-\tilde{e} \bar{v}) I\right)=1$. However, since $\tilde{e}<\bar{e}, r\left((-\Delta+K I)^{-1}(a+K-\tilde{e} \bar{v}) I\right)>1$. Hence we have a contradiction and the second possibility does not occur. This completes the proof.

Proof of Theorem 1. By Lemmas 1 and 2, there is a nondecreasing function $h$ defined on the interval $(\bar{c}, \mu)$ or $[\bar{c}, \mu]$ and with values in the interval $(0, \bar{e})$ such that $(c, e) \in T^{+}$if $\bar{e}>e>h(c)$ and $\bar{c}<c<\mu(\bar{c}<c \leq \mu$ if $h(\mu)$ is defined) while $(c, e) \notin T^{+}$if $e<h(c)$ or $c>\mu(c \geq \mu$ if $\mu$ is not in the domain of $h$ ). Since $T^{+}$is closed by Lemma 3, it follows that $(c, h(c)) \in T^{+}$if $c<\mu$ (or $c \leq \mu$ if $\mu$ is in the domain of $h$ ). Assume $(\tilde{c}, \tilde{e}) \in T^{+}$. If $\bar{c}<r<\tilde{c},(r, \tilde{e}) \in T^{+}$and, by Lemma 1 and its proof, 
there is an isolated strictly positive solution of $(\tilde{u}, \tilde{v})$ of (2) for $c=r$ and $e=\tilde{e}$ for which $\operatorname{index}_{D}(A,(\tilde{u}, \tilde{v}))=1$. By the homotopy invariance of the degree there is a nonnegative (and hence a strictly positive) fixed point of $A$ near $(\tilde{u}, \tilde{v})$ for each $(c, e)$ near $(r, \tilde{e})$. Hence $(r, e) \in \operatorname{int} T^{+}$. In particular, $(r, h(c)) \in \operatorname{int} T^{+}$if $\bar{c}<r<c$. Thus $h$ is strictly increasing. Similarly, if $(c, h(c)) \in T^{+},(c, s) \in \operatorname{int} T^{+}$for $h(c)<s<\bar{e}$. This ensures that $\mu$ is not in the domain of $h$ and $h$ is right continuous at $c$. (Remember that $h$ is increasing.) Since $h$ is increasing, one easily sees that the closedness of $T^{+}$ ensures that $h$ is left continuous at $c$. Hence $h$ is continuous. Nearly all the rest of Theorem 1 follows from Lemma 1 (with $f^{+}=h$ ). The only exception is to prove that there is a strictly positive solution if $e=\bar{e}$ and $\bar{c}<c<\mu$.

To prove this, note that the proof of Lemma 1 shows that if $\left(u_{1}, v_{1}\right)$ is a strictly positive solution for $e=f^{+}(c)$, then for each $e$ between $\left(f^{+}(c)\right.$ and $\bar{e}$ ) there is a strictly positive solution not in $C$. This follows because the sum of the indices of the strictly positive solutions in $C$ is 1 (counted with respect to $C$ or $D$ ) while the sum of the strictly positive solutions is zero. In particular there is a strictly positive solution $\left(u_{n}, v_{n}\right)$ not in $C$ if $e=\bar{e}-n^{-1}$. By choosing a subsequence if necessary, we can ensure that a subsequence of $\left(u_{n}, v_{n}\right)$ converges to a nonnegative solution of $(\tilde{u}, \tilde{v})$ of $(2)$ for $e=\bar{e}$. It remains to prove that $(\tilde{u}, \tilde{v})$ is a strictly positive solution. Thus we must show that it is impossible that $\left(u_{n}, v_{n}\right) \rightarrow(0,0)$ or $(\bar{u}, 0)$ or $(0, \bar{v})$. We prove that the last two cannot occur. The first is much easier. Since $c>\bar{c}$, the proof that the third possibility cannot occur is very similar to the proof of Lemma 3. Thus we may suppose that $\left(u_{n}, v_{n}\right) \rightarrow(\bar{u}, 0)$ in $E$ as $n \rightarrow \infty$. By using the regularity theory for $-\Delta$, we see that $u_{n} \rightarrow \bar{u}$ and $v_{n} \rightarrow 0$ in $C^{1}(\bar{\Omega})$ as $n \rightarrow \infty$. Now $u_{1}(x)<\bar{u}(x)$ on $\Omega$ and $\frac{\partial u}{\partial n}<\frac{\partial \bar{u}}{\partial n}$ on $\partial \Omega$. (To see the last property, we see easily that $u_{1}$ is a subsolution for the equation for $\bar{u}$ and we can apply the boundary point version of the maximum principle to the equation for $\bar{u}-u_{1}$.) Hence, for $n$ large, $u_{n} \geq u_{1}$. Similarly (but more easily), $v_{n} \leq v_{1}$ for $n$ large. Thus $\left(u_{n}, v_{n}\right) \geq_{S}\left(u_{1}, v_{1}\right)$ for $n$ large. Since $u_{n} \leq \bar{u},\left(u_{n}, v_{n}\right) \leq_{S}(\bar{u}, 0)$. Thus $\left(u_{n}, v_{n}\right) \in C$ for $n$ large. This gives a contradiction and our result is proved.

Remark. We have used in the last part of the proof that $\partial \Omega$ is smooth. This can be avoided by using linear operator theory ideas as in $\S 1$ of [10].

\section{NONEMPTINESS OF $T^{+}$AND $T^{-}$}

In this section, we discuss when $T^{+}$and $T^{-}$are nonempty and some estimates for them. In particular, we prove that at least one of them is nonempty for almost all $(a, d)$ with $a, d>\lambda_{1}$.

We first fix $c$ and increase $e$ across $\bar{e}$. We consider how strictly positive solutions bifurcate from $(\bar{u}, 0)$. If $e<\bar{e},(\bar{u}, 0)$ has index zero in $D$ while if $e>\bar{e},(\bar{u}, 0)$ has index 1 in $D$ (cf. part of the proof of Lemma 1 or p. 738 of [8]). Thus if we fix $a, d$, and $c$, a connected set of strictly positive solutions 
must bifurcate from $(\bar{u}, 0)$ into $D$ as $e$ increases across $\bar{e}$. To calculate the direction of a bifurcation, we use fairly standard bifurcation theory. We omit the details because it is almost the same as Cantrell and Cosner [4, §3]. The only difference is that we use $e$ as a parameter where they use $d$. (This has the effect of reversing their signs.) Then the conclusion is that small strictly positive solutions exist for all $e>\bar{e}$ but close to $\bar{e}$ if

$$
f_{1}(c) \equiv \int_{\Omega} h^{3}-\bar{e} c \int_{\Omega} h^{2} L(\bar{u} h)>0,
$$

while small strictly positive solutions exist for all $e<\bar{e}$ but close to $\bar{e}$ if $f_{1}(c)<0$. Here $h$ is the positive eigenfunction which spans the kernel of $-\Delta-(a-\bar{e} \bar{u}) I$ (for Dirichlet boundary conditions) and normalized so that $\|h\|_{2}=1$ and $L$ is the inverse of $-\Delta-(a-2 \bar{u}) I$ for Dirichlet boundary conditions. I should explain the argument a little. One looks for solutions of the form $(u, v)=(\bar{u}, 0)+s \tilde{h}+o(s)$ and $e(s)=\bar{e}+\beta s+\gamma s^{2}+o\left(s^{2}\right)$, where $\tilde{h}$ spans the kernel of $I-A^{\prime}(\bar{u}, 0)$ (and has second component positive in $\Omega$ ). It is easy to see that we only have nonnegative solutions for $s \geq 0$. One proves that $\beta=0$ and $\gamma$ has the same sign as $f_{1}(c)$. The claim then follows. Note that the test is indeterminate if $f_{1}(c)=0$. (We then need to look at higher order terms in the bifurcation equation.) It is shown in [4] that $L$ exists and $L$ is a positive operator. Hence we see that $\int h^{3}>0$ and $\int h^{2} L(\bar{u} h)>0$. Thus there is a $\tilde{c}>0$ such that $f_{1}(c)<0$ if $c>\tilde{c}$ while $f_{1}(c)>0$ if $c<\tilde{c}$. Note that $\tilde{c}<\bar{c}$ if $f_{1}(\bar{c})<0$ while $\tilde{c}>\bar{c}$ if $f_{1}(\bar{c})>0$.

Similarly, we define $f_{2}(e)=\int_{\Omega} k^{3}-\bar{c} e \int_{\Omega} k^{2} L_{2}(\bar{v} k)$, where $k$ is the positive function of $L^{2}$ norm 1 which spans the kernel of $-\Delta-(a-\bar{c} \bar{v}) I$ (with Dirichlet boundary conditions) and $L_{2}$ denotes the inverse of $-\Delta-(d-2 \bar{v}) I$ (for Dirichlet boundary conditions). Similarly to before, there is a positive $\tilde{e}$ with $f_{2}(\tilde{e})=0$. The main result of this section is the following one.

Theorem 2. (i) $T^{+}$is nonempty if either $f_{1}(\bar{c})>0$ or $f_{2}(\bar{e})<0$. Moreover, $\nu \leq \tilde{e}$ if $f_{2}(\bar{e})<0$ while $\mu \geq \tilde{c}$ if $f_{1}(\bar{c})>0$.

(ii) If $T^{+}$and $T^{-}$are both empty, then $f_{1}(\bar{c})=f_{2}(\bar{e})=0$.

(iii) For almost all $(a, d)$ in $\left(\lambda_{1}, \infty\right) \times\left(\lambda_{1}, \infty\right)$, either $T^{+}$is nonempty or $T^{-}$is nonempty.

Remark. We will later construct examples (with $\Omega$ star-shaped) for which $\tilde{c} \leq \bar{c}$ while $T^{+}$is nonempty and thus $\mu>\tilde{c}$, and where $\nu<\tilde{e}$ (in the latter case when $a<d$ ). Hence $\tilde{c}$ and $\tilde{e}$ do not always determine the limits of $T^{+}$. We will also construct an example where $f_{1}(\bar{c})$ and $f_{2}(\bar{e})$ have the same sign and thus $T^{+}$and $T^{-}$are both nonempty.

Proof of Theorem 2(i) and 2(ii). Suppose that $f_{1}(\bar{c})>0$. Hence, as we mentioned earlier, $\tilde{c}>\bar{c}$. Assume $\bar{c}<c_{1}<\tilde{c}$. Then $f_{1}\left(c_{1}\right)>0$. The branch of strictly positive solutions which branch at $\bar{e}$ bifurcates off for $e<\bar{e}$. Thus, if $\delta$ is small, (2) has a strictly positive solution if $c=c_{1}$ and $e=\bar{e}-\delta$. 
Thus $\left(c_{1}, \bar{e}-\delta\right) \in T^{+}$. Hence $T^{+}$is nonempty and, by Theorem $1, \mu>c_{1}$. Hence $\mu \geq \tilde{c}$. The other part of Theorem 2(i) is proved similarly. Theorem 2(ii) follows from Theorem 2(i) and its analogue for $T^{-}$.

We will prove Theorem 2(iii) by a series of lemmas.

Lemma 4. $f_{1}(\bar{c})$ and $f_{2}(\bar{e})$ are real analytic functions of $a$ and $d$.

Proof. By the implicit function theorem, we easily see that $\bar{u}$ is a real analytic function of $a$ (considered in $C_{0}(\bar{\Omega})$ ) and $\bar{v}$ is a real analytic function of $d$. (For example, in the first case, we apply the implicit function theorem to the equation $H(u, a)=0$ where $H(u, a)=-\Delta u-u(a-u)$ is considered as a map of $X=\left\{v \in W^{2, p}(\Omega): v=0\right.$ on $\left.\partial \Omega\right\} \times R$ to $L^{p}(\Omega)$ for $p>\frac{1}{2} m$. Hence $d-e \bar{u}$ is a real analytic function of $a, d, e$ (as a map into $C_{0}(\bar{\Omega})$.) Hence by Crandall and Rabinowitz [7, Lemma 1.3], the first eigenvalue $\tilde{\lambda}_{1}$ and corresponding normalized eigenfunction $\tilde{h}$ of $-\Delta+(d-e \bar{u}) I$ are real analytic functions of $a, d, e$. (We consider our equation as a mapping of $X$ into $L^{p}(\Omega)$.) By standard perturbation theory (cf. Kato [23, Theorem V.1.8 and $\S 2$ of Chapter 2])

$$
\frac{\partial \tilde{\lambda}_{1}}{\partial e}=-\int_{\Omega} \bar{u} h^{2}<0
$$

since $\bar{u}(x)>0$ in $\Omega$. Hence, by the implicit function theorem, we can solve the equation $\tilde{\lambda}_{1}(a, d, e)=0$ to obtain $e$ as a real analytic function of $(a, d)$. However, by the definition of $\bar{e}$, this solution is simply $\bar{e}$. Thus $\bar{e}$ is a real analytic function of $(a, d)$. Since $h=\tilde{h}(a, d, \bar{e})$, it follows that $h$ is a real analytic function of $(a, d)$. ( $h$ was defined at the start of this section.) Now the map $(d, e, w) \rightarrow-\Delta-(d-e w) I$ is a continuous polynomial map of $R \times R \times X \rightarrow \mathscr{B}\left(X, L^{P}\right)$ and hence is real analytic (where $\mathscr{B}(X, Y)$ denotes the bounded linear maps from $X$ to $Y$ ). Since the composite of real analytic maps is real analytic, it follows that the map $(a, d) \rightarrow-\Delta-(d-\bar{e} \bar{u}) I$ is real analytic. Since the operation of taking inverses is real analytic (because it has locally convergent power series expansions), it follows that the map $(a, d) \rightarrow L$ is real analytic (as a map into $\mathscr{B}\left(L^{P}, X\right)$ ). Since $\bar{c}$ is a real analytic function of $(a, d)$ by similar arguments, it now follows easily from the formula for $f_{1}(\bar{c})$ that $f_{1}(\bar{c})$ is a real analytic function of $(a, d)$. The proof for $f_{2}(\bar{e})$ is similar.

Remark. If $\Omega$ is not smooth, we have to work with slightly different spaces but the argument is still valid.

Lemma 5. If $\lambda_{1}<a$ and $d$ is large, $f_{1}(\bar{c})>0$ and $f_{2}(\bar{e})<0$.

We will prove this later in the section. Note that it can be proved that the first derivatives of $f_{1}(\bar{c})$ vanish on $d=a$ and hence we cannot use this obvious approach.

Proof of Theorem 2 (assuming Lemma 5). Now $f_{2}(\bar{e})$ is real analytic in $(a, d)$ and does not vanish identically (by Lemma 5). Hence (cf. Federer [17, 3.4.10]), 
$f_{2}(\bar{e}) \neq 0$ for almost all $(a, d) \in\left(\lambda_{1}, \infty\right) \times\left(\lambda_{1}, \infty\right)$. By Theorem 2(ii), this proves our claim.

Before proving Lemma 5 , it will be convenient to have some equalities and inequalities for $f_{1}(\bar{c})$ and $f_{2}(\bar{e})$. We first consider $f_{1}$. Now $w=L(\bar{u} h)$ is the solution of

$$
-\Delta w-(a-2 \bar{u}) w=\bar{u} h
$$

(with Dirichlet boundary conditions). However, by the equation for $h$,

$$
-\Delta h-(a-2 \bar{u}) h=(d-a) h+(2-\bar{e}) \bar{u} h
$$

(with the same boundary condition). Hence

$$
h=(d-a) L h+(2-\bar{e}) L(\bar{u} h) .
$$

Hence, if $\bar{e} \neq 2$, we see by substituting for $L(\bar{u} h)$ in the formula for $f_{1}(\bar{c})$ that

$$
f_{1}(\bar{c})=(2-\bar{e})^{-1}(2-\bar{e}-\bar{e} \bar{c}) \int_{\Omega} h^{3}+(\bar{e}-2)^{-1}(d-a) \bar{e} \bar{c} \int_{\Omega} h^{2} L h .
$$

Note that $h$ is positive and $L$ is a positive operator. In a moment, we will use this to prove part of Lemma 5 . It is possible to prove a slightly different inequality for $f_{1}(\bar{c})$ by using that $L(\bar{u} h) \leq a L h$ (since $\left.\|\bar{u}\|_{\infty} \leq a\right)$, hence using (13) to obtain an inequality for $L(\bar{u} h)$ and substituting this in the definition of $f_{1}(\bar{c})$.

We now obtain similar results for $f_{2}$. The analogue of (13) is

$$
k=-(d-a) L_{2} k+(2-\bar{c}) L_{2}(\bar{v} k)
$$

and the analogue of (14) is

$$
f_{2}(\bar{e})=(2-\bar{c})^{-1}(2-\bar{c}-\bar{e} \bar{c}) \int_{\Omega} k^{3}-(2-\bar{c})^{-1}(d-a) \bar{e} \bar{c} \int_{\Omega} k^{2} L_{2} k .
$$

It is easy to see that $f_{2}(\bar{e})<0$ if $\bar{c}<2,2-\bar{c}-\bar{e} \bar{c}<0$, and $d>a$. (Remember that $k>0$ in $\Omega$ and $L_{2}$ is a positive mapping.) Once again, we could obtain other inequalities for $f_{2}(\bar{e})$ by using that $L_{2}(\bar{v} k) \leq d L_{2} k$.

Proof of Lemma 5. We first estimate $\bar{c}$ and $\bar{e}$ (for $a$ fixed and $d$ large). Let $\tilde{v}=d^{-1} \bar{v}$. Then $\tilde{v}$ is a solution of

$$
-d^{-1} \Delta \tilde{v}=\tilde{v}(1-\tilde{v}) \quad \text { on } \Omega
$$

with $\tilde{v}=0$ on $\partial \Omega$. From the theory in $[12, \S 2], \tilde{v} \rightarrow 1$ in $L^{p}(\Omega)$ for each $p$ in $(1, \infty)$ as $d \rightarrow \infty$. Let $\hat{c}=d \bar{c}$. Now $r\left((-\Delta)^{-1}(a-\hat{c} \tilde{v}) I\right)=1$. Now, if $c>0, r\left((-\Delta)^{-1}(a-c \tilde{v}) I\right) \rightarrow r\left((-\Delta)^{-1}(a-c) I\right)=\lambda_{1}^{-1}(a-c)$ since $\tilde{v} \rightarrow 1$ in $L^{p}(\Omega)$ as $d \rightarrow \infty$ (cf. part of the proof that $r\left((-\Delta)^{-1}(a-c \bar{v}) I\right)<1$ for large $c)$. Thus, since $r\left((-\Delta)^{-1}(a-c \tilde{v}) I\right)$ is decreasing in $c$, it follows that $\lambda_{1}^{-1}(a-\hat{c})=1$, that is $\hat{c}=a-\lambda_{1}$. Hence $d \bar{c} \rightarrow a-\lambda_{1}$ as $d \rightarrow \infty$.

We now consider $\bar{e}$. Suppose $r>0$. We will prove that, if $d$ is large,

$$
r\left((-\Delta)^{-1}(d-r d \bar{u}) I\right)>1 \text {. }
$$


It follows that $\bar{e} \geq r d$ if $d$ is large. Since $r$ was arbitrary, it will follow that $d^{-1} \bar{e} \rightarrow \infty$ as $d \rightarrow \infty$. This will suffice for our purposes. To prove (16), it suffices to find $\mu_{d}>1$ and a nontrivial nonnegative function $s_{d}$ in $\dot{W}^{1,2}(\Omega)$ such that

$$
-\Delta s_{d} \leq \mu_{d}^{-1}(d-r d \bar{u}) s_{d}
$$

in the sense of distributions and such that $1-r \bar{u}(x)$ has a positive lower bound on $\left\{x \in \Omega: s_{d}(x) \neq 0\right\}$. (This follows because a simple calculation shows that this implies $(-\Delta+K I)^{-1}(d+K-r d \bar{u}) s_{d} \geq \alpha s_{d}$ for some $\alpha>1$ and then, by p. 265 in [29],

$$
r\left((-\Delta+K I)^{-1}(d+K-r d \bar{u}) I\right) \geq \alpha>1 .
$$

Here we choose $K>r d a$ to ensure the operator is positive. To prove (17), choose a neighbourhood $N$ of $\partial \Omega$ in $\Omega$ such that $u(x) \leq \frac{1}{2} r^{-1}$ on $N$. Let $w$ denote the first nonnegative eigenfunction of $-\Delta$ on $N$ (with Dirichlet boundary conditions on $\partial N$ ) and let $\tilde{\lambda}$ denote the corresponding eigenvalue. Define $s_{d}(x)$ to be $w(x)$ on $N$ and to be zero otherwise. Then $s_{d} \in \dot{W}^{1,2}(\Omega)$. Since $d-r d \bar{u}(x) \geq \frac{1}{2} d$ on $N$ (because $\bar{u}(x) \leq \frac{1}{2} r^{-1}$ on $N$ ), we easily see that $s_{d}$ pointwise satisfies (17) on $N$ if $d$ is large. In addition, (17) is trivially satisfied pointwise on $\Omega \backslash \bar{N}$. Hence we can deduce (17) on $\Omega$ by Berestycki and Lions [2, Lemma I.1]. This completes the proof that $d^{-1} \bar{e} \rightarrow \infty$ as $d \rightarrow \infty$.

It follows that $\bar{e} \bar{c} \rightarrow \infty$ as $d \rightarrow \infty$. Since $\bar{c} \rightarrow 0$ as $d \rightarrow \infty$, our comments after (15) now imply that $f_{2}(\bar{e})<0$ for large $d$. This proves the first part of Lemma 5.

Now consider $f_{1}$. By (14) and since $2-\bar{e}<0$ and $2-\bar{e}-\bar{e} \bar{c}<0$ for large $d$, we see that $f_{1}(\bar{c})>0$ if we can prove that

$$
\int_{\Omega} h^{3}>-(2-\bar{e}-\bar{e} \bar{c})^{-1}(d-a) \bar{c} \bar{e} \int_{\Omega} h^{2} L h
$$

for large $d$. Now, by our earlier asymptotics for $\bar{e}$ and $\bar{c}$, we easily see that

$$
(d-a) \bar{e} \bar{c} /(2-\bar{e}-\bar{e} \bar{c}) \sim \bar{e} d \bar{c} /(-\bar{e}) \rightarrow-\lambda_{1}
$$

as $d \rightarrow \infty$. Hence, it suffices to prove that, given $r>0$,

$$
\int_{\Omega} h^{2} L h<r \int_{\Omega} h^{3}
$$

for $d$ large. By Hölder's inequality,

$$
\int_{\Omega} h^{2} L h \leq\|h\|_{3}^{2}\|L h\|_{3} .
$$

Hence it suffices to prove that

$$
\|L h\|_{3} \leq r\|h\|_{3}
$$

for $d$ large. Note that $L$ is independent of $d$. By the usual $L^{p}-L^{q}$ estimates for $L$ (cf. [18, Theorems 7.10 and 8.15]), we see that there is a $q<3$ and 
a $K>0$ independent of $d$ or $h$ such that $\|L h\|_{3}<K\|h\|_{q}$. By Hölder's inequality, we can assume $q \geq 2$. Hence we have to prove that given $\tilde{r}>0$, $\|h\|_{q} \leq \tilde{r}\|h\|_{3}$ for $d$ large. It suffices to prove that $\|h\|_{3}$ is large for $d$ large because we can then obtain our estimate for $\|h\|_{q}$ if we note that $\|h\|_{2}=1$ and that $\|h\|_{q}^{q} \leq\|h\|_{3}^{2 \alpha}\|h\|_{3}^{3(1-\alpha)}$ where $2 \alpha+3(1-\alpha)=q$ by Hölder's inequality (cf. Theorem 13.19 in Hewitt and Stromberg [21]). In fact, it suffices to prove that, if $K$ is a compact subset of $\Omega$, then $\left\|\chi_{K} h\right\|_{2} \rightarrow 0$ as $d \rightarrow \infty$. This follows because this last inequality implies that the $L^{2}$ norm of $h$ on $\Omega \backslash K$ tends to 1 as $d \rightarrow \infty$. Since $\|h\|_{2, \Omega \backslash K} \leq m(\Omega \backslash K)^{1 / 6}\|h\|_{3, \Omega \backslash K}$ (with the obvious notation) by Hölder's inequality, it follows by choosing $K$ so that $m(\Omega \backslash K)$ is small that $\|h\|_{3, \Omega \backslash K}$ is large if $d$ is large and hence $\|h\|_{3}$ is large. Now, by the equation for $h$

$$
d^{-1} \int_{\Omega}|\nabla h|^{2}=\int_{\Omega}\left(1-\bar{e} d^{-1} \bar{u}\right) h^{2}
$$

Thus

$$
\int_{\Omega} \bar{u} h^{2} \leq d(\bar{e})^{-1} \int_{\Omega} h^{2} \rightarrow 0
$$

as $d \rightarrow \infty$ since $\int_{\Omega} h^{2}=1$ and $d^{-1} \bar{e} \rightarrow \infty$ as $d \rightarrow \infty$. If $K$ is a compact subset of $\Omega, \bar{u} \geq s>0$ on $K$ and hence we see that

$$
s \int_{K} h^{2} \leq \int_{\Omega} \bar{u} h^{2} \rightarrow 0
$$

as $d \rightarrow \infty$. Hence our claim follows. (Note that $\bar{u}$ is independent of $d$.) This completes the proof of Lemma 5 .

Remarks. 1. The above proof of Lemma 5 uses the smoothness of $\Omega$ and the selfadjointness of $-\Delta$. However, with more care these can both be avoided. In addition, we have used (to derive (14) and (15)) that we have the same linear part in both equations. This can also be avoided at least for $f_{2}$ for smooth domains at the expense of much more effort. (To prove the result for $f_{1}$ one would need better asymptotic estimates for $\bar{e}$ and $h$ as $d \rightarrow \infty$. This can probably be done. It can be done if $m=1$.)

2. Note that the equations are of the same type if $a$ and $d$ and $c$ and $e$ are simultaneously interchanged. Hence $f_{1}(\bar{e})(\tilde{a}, \tilde{d})=f_{2}(\bar{c})(\tilde{d}, \tilde{a})$, where $f_{1}(\bar{e})(\tilde{a}, \tilde{d})$ refers to the value of $f_{1}(\bar{e})$ for $a=\tilde{a}$ and $d=\tilde{d}$ and the other term is defined analogously. Thus Lemma 5 includes information for $a>d$. We do not need the full strength of the lemma to prove Theorem 2 but it is needed in $\S 3$.

3. By Lemma 5 and Theorem $1, T^{+}$is nonempty if $a>\lambda_{1}$ and $d$ is much larger than $a$.

4. We conjecture that $f_{2}(\bar{e})<0$ if $d>a$. On the other hand, we will give examples in $\S 3$ where $f_{1}(\bar{c})$ changes sign at points with $d>a$.

5. Note that we have used essentially in the proof of Lemma 5 that inf $\bar{u}=0$. 
Thus the proof of Lemma 5 does not apply for other boundary conditions. Indeed, one can show if the boundary conditions on the first equation are not Dirichlet, then $d^{-1} \bar{e} \rightarrow(\inf \bar{u})^{-1}$ as $d \rightarrow \infty$. (Here the boundary condition on the first equation is a Neumann or a Robin boundary condition $\frac{\partial u}{\partial n}=-b u$ where $b>0$ and $n$ is the outward normal.) Thus Lemma 5 does not apply for other boundary conditions. However, a more careful asymptotic analysis implies that Theorem 2 holds unless both boundary conditions are Neumann. Even if both equations have Neumann boundary conditions, it turns out that the analogue of Theorem 2 is true if we allow $c$ and $e$ to depend on $x$. More precisely, we replace $c$ by $c C(x)$ and $e$ by $e E(x)$ where $C$ and $E$ are positive continuous functions which are not constant functions. Thus the constant coefficient Neumann case where $T^{+}$and $T^{-}$are always empty is very much the exception. Indeed, it seems very likely that our methods can be generalized to prove that if $a$ and $d$ are functions of $x$, then $T^{+} \cup T^{-}$is nonempty in the Neumann case for 'most' functions $a(x)$ and $d(x)$. It is also possible to prove that the analogue of $T^{+}$and $T^{-}$may be nonempty for the time periodic Neumann problem in [6]. (One way is to use the asymptotic ideas in $\S 2$ of [9].)

6. Note that another way to see that $T^{+}$and $T^{-}$are empty for Neumann boundary conditions is to use the iteration in $\S 2$ of [9] and note that this iteration always gives constant functions (since the starting points are constants). Now by the theory in $\S 2$ of [9], if there is a strictly positive solution, this iteration must converge to a strictly positive solution. Hence there must be a constant strictly positive solution if there is a strictly positive solution. This is easily shown to be impossible. (This same idea is useful for studying the time dependent case discussed in [6].) As an alternative to the theory in [9], one can often start an iteration at points close to $(\bar{u}, 0)$ on the centre unstable manifold of $(\bar{u}, 0)$ (or of $(0, \bar{v}))$. Here we iterate by following the flow. This method has the advantage of applying more easily in degenerate situations but is more awkward when $\partial \Omega$ is not smooth. This is discussed further in $\S 4$.

7. We do not know whether our equation always has a strictly positive solution if $e=\bar{e}$ and $c=\bar{c}$. This is true in the Neumann case.

8. If $\nu<\tilde{e} \leq \bar{e}$, then it is not difficult to prove that our system has a strictly positive solution for $e=\nu$ and $c=\bar{c}$ (because one easily shows that solutions cannot bifurcate from $(\bar{u}, 0)$ as we decrease $e$ to $\nu)$. An analogous statement holds if $\mu>\tilde{c} \geq \bar{c}$.

9. As $d \rightarrow \infty$, one can prove with more care that $(\bar{e})^{-1} \tilde{e} \rightarrow 0$. Thus the closure of $T^{+}$may extend most of the way down $\{(c, e): c=\bar{c}, 0 \leq e \leq \bar{e}\}$.

Last, for this section, I want to briefly consider the implication of some of our ideas for uniqueness. Assume $\nu<\bar{e}$ and $\nu<\tilde{e}$. We will prove in the next section that this can occur. If $\nu<t<\tilde{e}$, then there is an $\varepsilon>0$ such that (2) has more than one strictly positive solution whenever $e=t$ and $\bar{c}-\varepsilon<c<\bar{c}$. (This is in a region where nonuniqueness was not known previously.) To see this, one chooses a strictly positive solution $\left(u_{1}, v_{1}\right)$ of $(2)$ for $c=\bar{c}$ and 
$e=t \quad(c>\bar{c}$ could also be used). By the proof of Lemma 2 of $\S 1$, there is an "asymptotically stable" strictly positive solution $(u, v)$ of (2) with $u \geq u_{1}$, $v \leq v_{1}$ for $c<\bar{c}$ and $e=t$. Since $u \geq u_{1}$, this solution does not have small first component. On the other hand, since $\nu<t<\tilde{e}$, the theory at the beginning of this section shows that a branch of strictly positive solutions bifurcates from $(0, \bar{v})$ as we decrease $c$ across $\bar{c}$ (for $e=t$ ) and this branch bifurcates into $c<\bar{c}$. Since these solutions have small first component, this proves our nonuniqueness claim. With a little more care, it can be shown that these bifurcating solutions are "asymptotically stable" and $\varepsilon$ can be chosen independent of $t$ on compact subsets of $(\nu, \tilde{e})$. Thus, if $\nu<\tilde{e} \leq \bar{e}$, we obtain an open subset of $(0, \bar{c}) \times(0, \bar{e})$ where there are at least two "asymptotically stable" strictly positive solutions. A simple degree argument now implies that there are at least three strictly positive solutions in this case.

Similarly, if $\mu>\tilde{c}$ and $\mu>\bar{c}$, a more careful use of some of our earlier ideas (especially Lemma 1 , the direction of bifurcation result in this section, and [9]) imply that if $\mu>t>\sup \{\tilde{c}, \bar{c}\}$ and $\varepsilon$ is small and positive, then (2) with $e=\bar{e}+\varepsilon$ and $c=t$ has at least three strictly positive solutions including at least one "asymptotically stable" solution. Note this gives points in $(\bar{c}, \infty) \times(\bar{e}, \infty)$ where there are "asymptotically stable" solutions. In this proof, we need to use that if we have an "asymptotically stable" solution for particular values of $c$ and $e$ and then we perturb $c$ and $e$ slightly, we must still have an "asymptotically stable" solution close by. In $\S 3$, we will construct an example where $\tilde{c} \leq \bar{c}<\mu$. It follows that, in this example, there exist $(c, e)$ in $(\bar{c}, \infty) \times(\bar{e}, \infty)$ arbitrarily close to $(\bar{c}, \bar{e})$ where there is an "asymptotically stable" strictly positive solution. (This contrasts with $\S 3$ of [12].) On the other hand, one can show that this behaviour does not occur when $a=d$. (One does this by first using some of the ideas in [6] to prove uniqueness in this range of parameters.)

Similar ideas can be used to prove that, if $T^{+}$and $T^{-}$are both nonempty, we can perturb $a$ and $d$ slightly to obtain an example where either (i) (2) has an asymptotically stable strictly positive solution for all $(c, e)$ near $(\bar{c}, \bar{e})$ or (ii) the strictly positive solution of (2) is not unique for every $(c, e)$ near $(\bar{c}, \bar{e})$ in $[0, \bar{c}) \times[0, \bar{e})$.

There are two last comments I would like to make on uniqueness. Assume that $a, d, c$ are fixed and $e$ is small. An easy perturbation analysis shows that, in this case, (2) has at most one strictly positive solution. A similar result holds if $a, d, e$ are fixed and $c$ is small or if $a, d$ are fixed and both $c$ and $e$ are small. This is related to work in [6]. Second, one does not always expect uniqueness for $c>\bar{c}$ and $e>\bar{e}$ even if $a=d, c=e$, and $\Omega$ is convex. If $\Omega$ is a ball, one can prove that a second radially symmetric strictly positive solution (and also nonradially symmetric ones) bifurcate off the one with $u=v$ as we vary $c$ (with $c=e$ ). An example of nonuniqueness with $\Omega$ star-shaped can also be obtained by the method of [14, p. 147]. These results give a negative answer to a question in [6]. 
Lastly for this section, the ideas in $\S 3$ of [13] can be used to prove that $|\mu-\bar{c}|+|\nu-\bar{e}|$ is $o(|d-a|)$ as $(a, d) \rightarrow(r, r)$ where $r>\lambda_{1}$. Thus $T^{+}$, when it exists will be very small when $d$ is close to $a$. Note that Theorem 2 implies that there must be $(d, a)$ near $(r, r)$ for which $T^{+}$or $T^{-}$is nonempty. This means that one of the comments near the bottom of p. 436 of [13] is not quite correct. (This occurs when the dominating term of the bifurcation equation in [13] vanishes identically.) Lastly, the methods in [13] give, for any domain $\Omega$, examples where $e>\bar{e}$ and $c>\bar{c}$ and there is a unique (necessary unstable) strictly positive solution.

\section{COUNTEREXAMPLES, UNIQUENESS, AND DOMAIN PERTURBATION}

In this section, we use our domain perturbation techniques from [14] to prove that a number of the possibilities in the last two sections can actually occur for star-shaped $\Omega$ if $m>1$. We also present two different types of examples where $(\bar{u}, 0)$ and $(0, \bar{v})$ are both unstable (that is $c<\bar{c}$ and $e<\bar{e}$ ) but (2) has more than one strictly positive solution. These seem to be the first such examples. In particular, we present such an example when $d$ is much larger than $a$ and an example where $d$ is close to $a$ and neither is large. We also obtain a number of other counterexamples. We always assume that $m>1$.

We first obtain examples of nonuniqueness with $d$ much larger than $a$. It is convenient, in order to quote the theory in [9], to use the form of the equations in [9], that is, without rescaling. More particularly, we consider the equations

$$
-\Delta u=u(a-b u-c v), \quad-\Delta v=s^{-1} v(e-f u-g v)
$$

with Dirichlet boundary conditions. Note that we use $s$ where $d$ is used in [9]. Note that the $d$ of the present paper is $s^{-1} e$. Thus $d$ large corresponds to $s$ small. By the theory in $\S 2$ of [9] (especially pp. 246 and 247), we see that we will have an example of nonuniqueness if we can find an $\Omega$ such that $a-c g^{-1} e<\lambda_{1}(\Omega)$ and the equation

$$
\begin{aligned}
-\Delta u & =u\left(a-b u-c g^{-1}(e-f u)^{+}\right) \text {in } \Omega, \\
u & =0 \text { on } \partial \Omega
\end{aligned}
$$

has at least two isolated sets of positive solutions of nonzero index in the natural cone $K_{1}$ in $C_{0}(\bar{\Omega})$. Here $\lambda_{1}(\Omega)$ is the first eigenvalue of $-\Delta$ on $\Omega$ for Dirichlet boundary conditions. A couple of points need to be made here. First, the examples are with $s$ small, and the solutions we obtain are close to $\left(u, g^{-1}(e-f u)^{+}\right)$where $u$ is a positive solution of $(18)$, and hence the Dirichlet boundary conditions ensure that neither component can vanish identically. Second, a slight variant of the argument on p. 247 of [9] ensures that

$$
r\left((-\Delta+K I)^{-1}(a-c \bar{v}+K) I\right)<1
$$

and

$$
r\left((-\Delta+K I)^{-1} s^{-1}(e-f \bar{u}+s K) I\right)>1
$$


for $s$ small and thus we are in the claimed part of the $(c, e)$ plane (in the notation of this paper).

Hence it suffices to construct the claimed example for (18). We first choose $\Omega$ to be the unit ball $B_{1}$ and choose $b<c g^{-1} f$. This ensures that as we decrease $a$ from $\lambda_{1}\left(B_{1}\right)+c g^{-1} e$, a branch of small positive solutions branches from the trivial solutions. A standard bifurcation theory argument ensures that, if $a$ is close to $\lambda_{1}\left(B_{1}\right)+c g^{-1} e$, this small positive solution $u_{1}(a)$ has invertible linearization. Theorem 1 in [11] ensures that the solution $u_{1}(a)$ has index \pm 1 (in fact -1 ) in the cone $K_{1}$. Here we use the map $A_{1}: K_{1} \rightarrow K_{1}$ defined by

$$
A_{1}(u)=(-\Delta+K I)^{-1}\left(u\left(a+K-b u-c g^{-1}(e-f u)^{+}\right)\right) .
$$

As in [9, p. 247], we see that if $a<\lambda_{1}(b)+c g^{-1} e$, the sum of the indices of the positive solutions of (18) in $K_{1}$ is zero. Hence, if $W_{a}$ denotes the set of positive solutions of (18) in $K_{1}$ except $u_{1}(a)$, then $\operatorname{index}_{K_{1}}\left(A_{1}, W_{a}\right)= \pm 1$. Note that the above argument does not assume that $W_{a}$ consists only of isolated solutions. We now choose a second ball $\bar{x}+B_{r}$ so that the two open balls do not intersect but touch and such that $a>\lambda_{1}\left(B_{r}+\bar{x}\right)+c g^{-1} e$. This holds if $a>\lambda_{1}\left(B_{1}\right) r^{-2}+c g^{-1} e$ since $\lambda_{1}\left(B_{r}+\bar{x}\right)=r^{-2} \lambda_{1}\left(B_{1}\right)$. This last result follows by a simple scaling. Since $a>\lambda_{1}\left(B_{r}+\bar{x}\right)+c^{-1} g e$, the theory in [9] ensures that the sum of the indices of the positive solutions of (18) is 1 . Let $Z$ denote this set of positive solutions. Note our condition on $a$ ensures that zero is an isolated nonnegative solution. Now consider $(18)$ on $\Omega=B_{1}(0) \cup\left(B_{r}+\bar{x}\right)$. Here we look for solutions in $\dot{W}^{1,2}(\Omega) \cap L^{\infty}(\Omega)$. As in [14, pp. 128-129], we see that $u$ is a solution of (18) on $\Omega$ (including the weak boundary condition) if and only if $u$ is a solution on $B_{1}(0)$ and $B_{r}+\bar{x}$. Moreover, by the product theorem for the degree, the index of the set of solutions $\left\{u_{1}(\alpha)\right\} \times Z$ (of $A_{1}$ on $B_{1}(0) \cup\left(B_{r}+\bar{x}\right)$ in the cone $\left.K_{2}=K_{1} \oplus K_{1}\right)$ has index $K_{1}\left(A_{1}, u_{1}(\alpha)\right) \times \operatorname{index}_{K_{1}}\left(A_{1}, Z\right)=-1 \cdot 1=$ -1 . Here, in the first index $\Omega=B_{1}(0)$ while in the second $\Omega=B_{r}+\bar{x}$. Here we are implicitly using that the commutativity theory for the degree ensures that index $_{K_{1}}\left(A_{1}, Z\right)$ is the same in $\dot{W}^{1,2}(\Omega)$ or $C_{0}(\bar{\Omega})$ (or $L^{p}(\Omega)$ for large $p$ ). Choose $\Omega_{n}$ star-shaped for $n \geq 4$ such that $\Omega_{n}$ decreases to $B_{1}(0) \cup\left(B_{r}+\bar{x}\right)$ as $n \rightarrow \infty$. More precisely, we mean convergence in the sense of [15]. By a slight variant of Theorems 1 and 2 in [14], we see that for large $n$ the sum of the indices of the positive fixed points of $A_{1}$ in $L^{p}\left(\Omega_{n}\right)$ near $\left\{u_{a}\right\} \times Z$ is -1 . (The variant we use really only involves a slight modification of the proof of Step 2 of the proof of Theorem 1 in [14]. It is here that we use $m>1$.) Similarly, for large $n$, the sum of the indices of the positive fixed points of $A_{1}$ in $L^{p}\left(\Omega_{n}\right)$ near $W_{a} \times Z$ is +1 . In particular, we have two disjoint sets of nontrivial positive fixed points of $A_{1}$ each of which have nonzero index. By our earlier comments (and in particular by Proposition 1 in [9]) this provides our example of nonuniqueness. (It is an example with $\Omega=\Omega_{n}$ star-shaped and $d$ very large.) Note that rescaling our equations to fit them into the form 
studied in the bulk of this paper does not affect the example.

We now obtain a number of counterexamples with $d$ close to $a$. Later in this section, we will construct two disjoint set $\Omega_{1}, \Omega_{2}$ and $d$ close to $a$ such that $\bar{c}_{1}<\bar{c}_{2}$ and $\bar{e}_{2}<\bar{e}_{1}$ (with the obvious notation). Moreover, $\Omega_{1}$ and $\Omega_{2}$ are $C^{2}$ close to balls and hence are strongly convex. Assuming this for a moment, we will construct our counterexamples.

We can translate $\Omega_{2}$ so that $\Omega_{1}$ and $\Omega_{2}$ do not intersect but their closures intersect at a single point. Choose $\Omega_{n}$ star-shaped for $n \geq 4$ such that $\Omega_{n}$ decreases to $\widetilde{\Omega}=\Omega_{1} \cup \Omega_{2}$ in the sense of [15]. We will find formulae for $\bar{c}, \bar{e}, f_{1}(\bar{c})$, and $f_{2}(\bar{e})$ for $\widetilde{\Omega}$ and then show that those for $\Omega_{n}$ are close to those for $\widetilde{\Omega}$.

We first prove that $\bar{c}_{\widetilde{\Omega}}=\sup \left\{\bar{c}_{1}, \bar{c}_{2}\right\}$ and an analogous formula for $\bar{e}_{\widetilde{\Omega}}$. Here $\bar{c}_{\widetilde{\Omega}}$ denotes $\bar{c}$ for the set $\widetilde{\Omega}$. We define $\bar{v}_{\widetilde{\Omega}}(x)$ to be $\bar{v}_{\Omega_{1}}(x)$ on $\Omega_{1}$ and to be $\bar{v}_{\Omega_{2}}(x)$ on $\Omega_{2}$. A simple degree argument using Theorem 2 in [14] shows that this is the nontrivial nonnegative solution on $\widetilde{\Omega}$ which perturbs to give the nonunique trivial positive solution of

$$
-\Delta v=v(d-v) \quad \text { on } \Omega_{n}, \quad v=0 \text { on } \partial \Omega_{n} .
$$

Now the operator $(-\Delta+K I)^{-1}\left(K+a-c \bar{v}_{\widetilde{\Omega}}\right) I$ on $\widetilde{\Omega}$ is the direct sum of the corresponding operators on $\Omega_{1}$ and $\Omega_{2}$. Thus its spectral radius on $\widetilde{\Omega}$ is the larger of its spectral radii on $\Omega_{1}$ and $\Omega_{2}$. Hence this spectral radius is 1 precisely when both of the spectral radii on $\Omega_{1}$ and $\Omega_{2}$ are less than or equal to 1 and at least one is equal to 1 . Since the spectral radii on $\Omega_{1}$ and $\Omega_{2}$ decrease as $c$ increases, it follows that $\bar{c}_{\widetilde{\Omega}}=\sup \left\{\bar{c}_{1}, \bar{c}_{2}\right\}$. Similarly $\bar{e}_{\widetilde{\Omega}}=\sup \left\{\bar{e}_{1}, \bar{e}_{2}\right\}$. In addition, note that the unique nonnegative solution of $(-\Delta+K) k=\left(a+K-\bar{c}_{\widetilde{\Omega}} \bar{v}_{\widetilde{\Omega}}\right) k$ (on $\left.\widetilde{\Omega}\right)$ is $k_{\widetilde{\Omega}}$ where $k_{\widetilde{\Omega}}(x)$ is $k_{2}(x)$ on $\Omega_{2}$ and is zero otherwise. (Remember that $\bar{c}_{2}>\bar{c}_{1}$.) Here $k_{2}$ is the solution of $(-\Delta+K I) k=\left(a+K-\bar{c}_{2} \bar{v}_{\Omega_{2}}\right) k$ on $\Omega_{2}$ with $L^{2}$ norm 1 . An analogous result holds for $h_{\widetilde{\Omega}}$ except that $h_{\widetilde{\Omega}}$ is supported on $\Omega_{1}$.

Now assume $\Omega_{n}$ are star-shaped sets (for $n \geq 4$ ) which converge to $\Omega_{1} \cup \Omega_{2}$ in the sense of [15] and assume that $\bigcup_{n=4}^{\infty} \Omega_{n} \cup \Omega_{1} \cup \Omega_{2} \subseteq B$. Then, as in the proof of Theorems 1 and 2 in [14], one easily sees that $\bar{v}_{\Omega_{n}} \rightarrow \bar{v}_{\widetilde{\Omega}}$ and $\bar{u}_{\Omega_{n}} \rightarrow \bar{u}_{\widetilde{\Omega}}$ in $L^{p}(B)$ for all $p, \bar{c}_{\Omega_{n}} \rightarrow \bar{c}_{\widetilde{\Omega}}=\sup \left\{\bar{c}, \bar{c}_{2}\right\}$, and $\bar{e}_{\Omega_{1}} \rightarrow \bar{e}_{\widetilde{\Omega}}=\sup \left\{\bar{e}_{1}, \bar{e}_{2}\right\}$ as $n \rightarrow \infty$. Moreover $k_{\Omega_{n}}$ (normalized to have $L^{2}$ norm 1$) \rightarrow k_{\widetilde{\Omega}}$ in $L^{p}(B)$ as $n \rightarrow \infty$ and $k_{\Omega_{n}}$ are uniformly bounded in $L^{\infty}$. A similar result holds for $h_{\Omega_{n}}$.

We now construct some of our examples. Suppose that $c>\bar{c}_{2}$ and $\bar{e}_{2}<e<$ $\bar{e}_{1}$. Thus $c>\bar{c}_{\Omega_{n}}$ and $e<\bar{e}_{\Omega_{n}}$ for large $n$. Since $c>\bar{c}_{1}$, the solution $\left(0, \bar{v}_{1}\right)$ of (2) on $\Omega_{1}$ has nonzero index in the cone $K_{2}$ (for $\Omega=\Omega_{1}$ ). Similarly, since $\bar{e}_{2}<e$ the solution $\left(\bar{u}_{2}, 0\right)$ of $(2)$ has nonzero index in $K_{2}$ for $\Omega=\Omega_{2}$. 
We can now use similar arguments to those in the first example to deduce that, for $n$ large, there is a solution $\left(u_{n}, v_{n}\right)$ of (2) in $K_{2}$ for $\Omega=\Omega_{n}$ near $\left(\tilde{u}_{1}, \tilde{v}\right)$ in $L^{p}(B) \times L^{p}(B)$ where $\tilde{u}(x)=\bar{u}_{2}(x)$ if $x \in \Omega_{2}$ and is zero otherwise, and $\tilde{v}(x)=\bar{v}_{1}(x)$ if $x \in \Omega_{1}$ and is zero otherwise. (This is also similar to $\mathrm{p}$. 147 of [14].) Since $\tilde{u}$ and $\tilde{v}$ do not vanish identically, $\left(u_{n}, v_{n}\right)$ is a strictly positive solution. Since $c>\bar{c}_{\Omega_{n}}$ and $e<\bar{e}_{\Omega_{n}}$, it follows that $(c, e) \in T_{n}^{+}$for $n$ large. Here $T_{n}^{+}$is $T^{+}$for $\Omega=\Omega_{n}$. Hence $T_{n}^{+}$is nonempty for large $n$. Since we can choose $c$ large, we also see that $\mu_{n} \rightarrow \infty$ as $n \rightarrow \infty$ where our notation follows that of $\S 2$ except that $\mu_{n}$ is $\mu$ for $\Omega=\Omega_{n}$. On the other hand, by using the definition of $\tilde{c}$ and our convergence results, we easily see that $\tilde{c}_{n}$ (that is $\tilde{c}$ for $\Omega=\Omega_{n}$ ) tends to $\tilde{c}_{\tilde{\Omega}}=\tilde{c}_{1}$ as $n \rightarrow \infty$. Hence we have examples of sets where $\tilde{c}<\mu$ (and $\mu>\bar{c}$ ). Second, since we can use a similar argument if $\bar{c}_{1}<c<\bar{c}_{2}$ and $e>\bar{e}_{1}$, we have examples where $T^{+}$and $T^{-}$ are both nonempty.

Next we show that, if either $T_{2}^{-}$or $T_{1}^{+}$is nonempty, then the above ideas give examples where $c<\bar{c}$ and $e<\bar{e}$ but there is more than one strictly positive solution. To do this, we assume $T_{1}^{+}$is nonempty. The other case is simpler. Choose $(c, e) \in \operatorname{int} T_{1}^{+}$so that $\bar{c}_{1}<c<\bar{c}_{2}$ and $\bar{e}_{2}<e<\bar{e}_{1}$. Note that Theorem 1 and our assumptions that $\bar{c}_{1}<\bar{c}_{2}$ and $\bar{e}_{2}<\bar{e}_{1}$ ensure that we can do this. By Theorem 1, there is an "asymptotically stable" strictly positive solution $(\hat{u}, \hat{v})$ on $\Omega_{1}$. (Thus it has nonzero index in $K_{2}$.) We can now argue much as before and show that for $n$ large there is a strictly positive solution near $\left(\hat{u}_{1}, v_{1}\right)$ in $L^{p}(B) \times L^{p}(B)$ and one near $(\tilde{u}, \tilde{v})$. Here $v_{1}(x)=\bar{v}_{2}(x)$ on $\Omega_{2}$ and is zero otherwise, $\hat{v}_{1}$ is defined to be $\bar{u}_{1}$ on $\Omega_{1}$ and to be zero otherwise, and

$$
\hat{u}(x)=\left\{\begin{array}{ll}
\hat{u}(x) & \text { on } \Omega_{1}, \\
0 & \text { on } \Omega_{2},
\end{array} \quad \text { while } \tilde{v}(x)= \begin{cases}\hat{v}(x) & \text { on } \Omega_{1}, \\
\bar{v}_{2} & \text { on } \Omega_{2} .\end{cases}\right.
$$

Thus we have nonuniqueness. Note that $\bar{c}_{\Omega n} \rightarrow \bar{c}_{2}$ and $\bar{e}_{\Omega n} \rightarrow \bar{e}_{1}$ as $n \rightarrow \infty$ and hence we are in the parameter range we claimed. In fact, with a little more care one can deduce that $\left(\hat{u}_{1}, v_{1}\right)$ and $(\tilde{u}, \tilde{v})$ are both "asymptotically stable" and thus there are "asymptotically stable" solutions near $\left(\hat{u}_{1}, v_{1}\right)$ and $(\tilde{u}, \tilde{v})$ (on $\Omega_{n}$ ) for $n$ large. Thus, we have two "asymptotically stable" solutions. It remains to remove our condition that either $T_{2}^{-}$or $T_{1}^{+}$is nonempty. As we will see a little later, we can find three sets $\Omega_{1}, \Omega_{2}, \Omega_{3} C^{2}$ close to a ball such that $\bar{c}_{1}<\bar{c}_{2}<\bar{c}_{3}$ and $\bar{e}_{1}>\bar{e}_{2}>\bar{e}_{3}$. By varying $a$ and $d$ slightly and by using Theorem 2, we see that we can assume that either $T_{2}^{+}$is nonempty or $T_{2}^{-}$is nonempty. In the latter case, we can apply our argument earlier in the paragraph to $\Omega_{1}, \Omega_{2}$ while in the former case we can apply our argument to $\Omega_{2}, \Omega_{3}$. Thus, in all cases, we have the required example.

To complete the construction of the above examples, we need to construct $\bar{c}_{i}$ and $\bar{e}_{i}$ with the required properties. To do this, first note that, when $a=d$, $\bar{u}=\bar{v}, \bar{c}=\bar{e}=1$, and $h$ and $k$ are multiples of $\bar{u}$. Since $\bar{c}=1$ on the 
line $a=d$, we see that $\bar{c}_{, d}=-\bar{c}_{, a}$ when $a=d$, where $\bar{c}_{, d}$ denotes the partial derivative with respect to $d$ of $\bar{c}$, and $\bar{c}_{, a}$ is defined analogously. By differentiating the equation $-\Delta k=(a-\bar{c} \bar{v}) k$ with respect to $a$ and taking the scalar product with respect to $\bar{u}$, we easily see that $\bar{c}_{, a}=\left(\int_{\Omega} \bar{u}^{3}\right)^{-1} \int_{\Omega} \bar{u}^{2}$ when $a=d$. Similarly, $\bar{e}_{, d}=\left(\int_{\bar{\Omega}} \bar{u}^{3}\right)^{-1} \int_{\Omega} \bar{u}^{2}$. (For future reference, note that it follows easily that $(\bar{e} \bar{c})_{, a}=0$ when $d=a$.) If we increase $d$ slightly while keeping $a$ fixed, $\bar{c} \approx 1-\left.(d-a) \bar{c}_{, a}\right|_{d=a}$ while $\bar{e} \approx 1+\left.(d-a) \bar{c}_{, a}\right|_{d=a}$ (since $\bar{c}_{, a}=-\bar{e}_{, a}$ when $d=a$ ). Thus, we can obtain our required examples if we find three strongly convex domains $\Omega_{1}, \Omega_{2}, \Omega_{3}$ with $\left.\bar{c}_{, a}\right|_{d=a}$ all different. Thus, it suffices to find $\Omega_{1}, \Omega_{2}, \Omega_{3}$ as above with $\left(\int_{\Omega} \bar{u}^{3}\right)^{-1} \int_{\Omega} \bar{u}^{2}$ all different. Now it is easy to see that this expression depends continuously on $\Omega$ as $\Omega$ is changed smoothly (by Theorem 2 in [14], though it can be proved more easily). We consider dilations $\lambda \Omega$ of a fixed $\Omega$ with $0 \in \Omega$. It suffices to show our expression is nonconstant in $\lambda$. Now $\int_{\Omega} \bar{u}^{3} \leq\|\bar{u}\|_{\infty} \int \bar{u}^{2}$. Thus, our expression becomes large if $\|\bar{u}\|_{\infty}$ is small. This must occur because $\bar{u}$ bifurcates from zero as $\lambda_{1}(\lambda \Omega)$ crosses $a$. (Equation (2) has only the trivial nonnegative solution with $v$ vanishing identically if $a \leq \lambda_{1}(\lambda \Omega)$.) This completes the construction of the above examples.

We use our techniques to produce an example with $\bar{c}<\bar{c}$ or equivalently that $f_{1}(\bar{c})>0$. Since $f_{1}(\tilde{c})=0$, we see easily from the definition of $f_{1}(c)$ and $\tilde{c}$ that

$$
f_{1}(\bar{c})=(\tilde{c}-\bar{c}) \int_{\Omega} h^{2} L(\bar{u} h) .
$$

By differentiating this in $d$ and letting $d=a$, we see after a simple calculation that $\left(\tilde{c}_{, d}-\bar{c}_{, d}\right) \int_{\Omega}(\bar{u})^{3}\left(\int_{\Omega} \bar{u}^{2}\right)^{-3 / 2}=\left.\left(f_{1}(\bar{c})\right)_{, d}\right|_{d=a}$. Here we have used that $\tilde{c}=\bar{c}=1$, that $h$ is a multiple of $\bar{u}$ when $d=a$, and that $L\left(\bar{u}^{2}\right)=\bar{u}$ (by the equation for $\bar{u}$ ). (Note that $\tilde{c}=1$ since $T^{+}$and $T^{-}$are both empty when $a=d$.) Thus $\bar{c}_{, d}=\tilde{c}_{, d}$ when $a=d$ if we prove that $\left(f_{1}(\bar{c})\right)_{, d}$ is zero when $a=d$. To prove this, we use (13). If we use this, that $\bar{c}=\bar{e}=1$ when $a=d$, that $L^{-1}\left(\bar{u}^{2}\right)=\bar{u}$, and use our formulae for $\bar{c}_{, d}$ and $\bar{e}_{, d}$, we find after a tedious calculation that $\left(f_{1}(\bar{c})\right)_{, d}=0$ when $a=d$ and thus $\bar{c}_{, d}=\tilde{c}_{, d}$ when $a=d$. We now consider our earlier construction of an example with $\bar{c}_{2}>\bar{c}_{1}$. We did this by constructing $\Omega_{1}, \Omega_{2}$ such that $\left(\bar{c}_{2}\right)_{, d}>\left(\bar{c}_{1}\right)_{, d}$ when $d=a$. Hence, $\left(\tilde{c}_{2}\right)_{, d}>\left(\bar{c}_{1}\right)_{, d}$. Hence if we increase $d$ slightly keeping $a$ fixed, we see that $\tilde{c}_{2}>\bar{c}_{1}$ and $\bar{c}_{2}>\bar{c}_{1}$. If $\Omega_{n}$ decrease to $\Omega_{1} \cup \Omega_{2}$ as before, we see as before that $\bar{c}_{\Omega n} \rightarrow \bar{c}_{2}$ and $\tilde{c}_{n} \rightarrow \tilde{c}_{1}$. (Remember that $\bar{e}_{1}>\bar{e}_{2}$.) Thus, for $n$ large, $\tilde{c}_{n}<\bar{c}_{\Omega n}$ which proves our claim.

Lastly for this section, we produce an example where $\inf \left\{c:(c, e) \in T^{-}\right\}<$ $\tilde{c}$. (By interchanging $a$ and $d$ and $c$ and $e$, this produces an example where $\nu<\tilde{e}$.) We fix $a$ and let $d$ tend to infinity. Now $d \bar{c} \rightarrow a-\lambda_{1}(\Omega)$ as $d \rightarrow \infty$ (cf. the proof of Lemma 5). Hence if we choose two convex $\Omega$ 's, $\Omega_{1}, \Omega_{2}$, with $\lambda_{1}\left(\Omega_{2}\right)<\lambda_{1}\left(\Omega_{1}\right)$, then $\bar{c}_{2}>\bar{c}_{1}$ if $d$ is large. Now, if $d$ is large, we proved in 
$\S 2$ that $\tilde{c}_{1}>\bar{c}_{1}$ and $\tilde{c}_{2}>\bar{c}_{2}$. We will prove that if we choose $\Omega_{n}$ as before then $\liminf _{n \rightarrow \infty} \tilde{c}_{n} \geq \inf \left\{\tilde{c}_{1}, \tilde{c}_{2}\right\}>\bar{c}_{1}$. On the other hand, by arguing as earlier, we easily see that, if $\bar{c}_{1}<c<\bar{c}_{2}$ and $e>\sup \left\{\bar{e}_{1}, \bar{e}_{2}\right\}$, then $(c, e) \in T_{n}^{-}$for large $n$. Thus, $\lim \sup _{n \rightarrow \infty} \inf \left\{c:(c, e) \in T_{n}^{-}\right\} \leq \bar{c}_{1}$ and hence, for large $n, \inf \left\{c:(c, e) \in T_{n}^{-}\right\}<\inf \left\{\tilde{c}_{1}, \tilde{c}_{2}\right\}$ and thus $\inf \left\{c:(c, e) \in T_{n}^{-}\right\}<\tilde{c}_{n}$ for large $n$. This is our required counterexample. It remains to prove that $\liminf \operatorname{in}_{n \rightarrow \infty} \tilde{c}_{n} \geq \inf \left\{\tilde{c}_{1}, \tilde{c}_{2}\right\}$. It suffices to prove that, on $\Omega_{n}, f_{1, n}(c)>0$ if $0 \leq c<\inf \left\{\tilde{c}_{1}, \tilde{c}_{2}\right\}$ for $n$ large. Here $f_{1, n}$ is $f_{1}$ in $\Omega_{n}$. Remember that $f_{1, n}(c)$ decreases in $c$. We prove this result in the case where $\bar{e}_{1}=\bar{e}_{2}$. The other cases are easier. Now

$$
-\Delta h_{n}=\left(d-\bar{e}_{n} \bar{u}_{n}\right) h_{n}
$$

where $\left\|h_{n}\right\|_{2}=1$. By a similar argument to that in the proof of Step 1 of the proof of Theorem 1 in [14], a subsequence of $h_{n}$ converges weakly in $\dot{W}^{1,2}(\widetilde{B})$ and strongly in $L^{2}(\widetilde{B})$ to $\tilde{h} \in \dot{W}^{1,2}\left(\Omega_{1} \cup \Omega_{2}\right)$ where $-\Delta \tilde{h}=$ $\left(d-\bar{e}_{\Omega_{1} \cup \Omega_{2}} \bar{u}_{\Omega_{1} \cup \Omega_{2}}\right) \tilde{h}$. Since $\left\|h_{n}\right\|_{2}=1,\|\tilde{h}\|_{2}=1$. By the same arguments as in [14], we see that $\left.\tilde{h}\right|_{\Omega_{1}} \in \dot{W}^{1,2}\left(\Omega_{1}\right)$ and $\left.\tilde{h}\right|_{\Omega_{2}} \in \dot{W}^{1,2}\left(\Omega_{2}\right)$. Since $\bar{e}_{\Omega_{1} \cup \Omega_{2}}=$ $\bar{e}_{1}=\bar{e}_{2}$ and $\left.\bar{u}_{\Omega_{1} \cup \Omega_{2}}\right|_{\Omega_{1}}=\bar{u}_{1}$ (with an analogous result on $\Omega_{2}$ ), we see that $\left.\tilde{h}\right|_{\Omega_{1}}=\alpha h_{1}$. Since $\tilde{h} \geq 0, \alpha \geq 0$. Similarly $\left.\tilde{h}\right|_{\Omega_{2}}=\beta h_{2}$ where $\beta \geq 0$. Since $\|\tilde{h}\|_{2}=1, \alpha^{2}+\beta^{2}=1$. It is easy to see using the techniques of [14] and the formula for $f_{1}$ that, for fixed $c, f_{1, n}(c) \rightarrow f_{1, \Omega_{1} \cup \Omega_{2}}(c)$ as $n \rightarrow \infty$. Here we use the obvious notation and let the eigenfunction on $\Omega_{1} \cup \Omega_{2}$ be $\tilde{h}$. By splitting up the expression (equation (13)) for $f_{1}$ on $\Omega_{1} \cup \Omega_{2}$ to a part on $\Omega_{1}$ and a part on $\Omega_{2}$, we find that $f_{1, n}(c) \rightarrow \alpha^{3} f_{1,1}(c)+\beta^{3} f_{1,2}(c)$ as $n \rightarrow \infty$. Here $f_{1,1}$ denotes $f_{1}$ on $\Omega_{1}$ and $f_{1,2}$ is defined analogously. Now the right-hand side is positive if $c<\inf \left\{\tilde{c}_{1}, \tilde{c}_{2}\right\}$ (because $f_{1,1}(c)>0, f_{1,2}(c)>0, \alpha \geq 0$, $\left.\beta \geq 0, \alpha^{2}+\beta^{2}=1\right)$. This completes the proof.

We do not have an example where $d>a$ and $\nu<\tilde{e}<\bar{e}$. However, if the conjecture that $\tilde{e}<\bar{e}$ when $d>a$ is false, it seems very likely that our domain perturbation methods can be used to give an example.

\section{SOME REMARKS ON ASYMPTOTIC BEHAVIOUR}

In this section, we want to discuss rather briefly the implications of some of our ideas for the asymptotic behaviour of solutions of (1). Many of our ideas are implicit in [9] but here we emphasize their use more.

Assume that $c<\bar{c}$ and $\Omega$ is smooth enough so that the solution of the Dirichlet problem for $\Delta$ (and $L^{\infty}$ right-hand side) is continuous on $\bar{\Omega}$. Later on, we will see that either of these two assumptions can be relaxed. Assume that $\left(u_{0}, v_{0}\right) \in L^{\infty}(\Omega) \times L^{\infty}(\Omega)$, that $u_{0}$ and $v_{0}$ are nonnegative, and neither $u_{0}$ nor $v_{0}$ is equal a.e. to the zero function. Let $(u(t), v(t))$ denote the solution of (1) with initial values $\left(u_{0}, v_{0}\right)$. By the results in $\S 2$ of [9] there is a solution $(\tilde{u}, \tilde{v})$ of $(2)$ in $D$ other than $(0,0)$ such that every solution $(\hat{u}, \hat{v})$ of $(2)$ other 
than $(0, \bar{v})$ satisfies $(\tilde{u}, \tilde{v})<_{S}(\hat{u}, \hat{v})$. We prove that the $\omega$ limit set of the solution $(u(t), v(t))$ lies in $\left\{(z, y) \in D:(z, y) \geq_{S}(\tilde{u}, \tilde{v}), z \leq \bar{u}, y \leq \bar{v}\right\}$. In particular, it follows that, if $c<\bar{c}$ and $e>\bar{e}$ but $(c, e) \notin T^{-}$, then $(\tilde{u}, \tilde{v})=(\bar{u}, 0)$ and $(u(t), v(t)) \rightarrow(\bar{u}, 0)$ as $t \rightarrow \infty$. In this particular case, we have a complete picture of the dynamics. To prove our claim note that, in [9], a sequence $\left(u_{n}, v_{n}\right)$ was constructed such that $\left(u_{1}, v_{1}\right)=(0, \bar{v})$ and either $\left\{\left(u_{n}, v_{n}\right)\right\}$ increased to $(\tilde{u}, \tilde{v})$ (for the order $\left.\geq_{S}\right)$ or the sequence $\left\{\left(u_{n}, v_{n}\right)\right\}$ terminates and there is no strictly positive solution. Thus it suffices to prove for each $n$ that the omega limit set $\omega(u(t), v(t)) \geq_{S}\left(u_{n}, v_{n}\right)$. We prove this inductively. (If the sequence terminates, we show that $\omega((u(t), v(t)))=(\bar{u}, 0)$.) By the first equation of (1),

$$
\frac{\partial u(t)}{\partial t}-\Delta u(t) \leq u(t)(a-u(t))
$$

Now by a standard argument (cf. [5]), the solution $\hat{u}(x, t)$ of

$$
\frac{\partial u}{\partial t}-\Delta u=u(a-u), \quad u(x, 0)=u_{0}
$$

converges to $\bar{u}$ in $C_{0}(\bar{\Omega})$ as $t \rightarrow \infty$. (One proves that the solution converges in $L^{2}$ and is compact in $C_{0}(\bar{\Omega})$.) By the parabolic maximum principle (cf. [28, Chapter 3]) $u(x, t) \leq \hat{u}(x, t)$. Thus $\omega(u(t), v(t)) \subseteq\{(z, y): z \leq \bar{u}\}$. By the same argument, we can establish the corresponding result for $v(t)$. Thus $u(x, t) \leq \bar{u}(x)+\varepsilon$ for large $t$. Suppose we can prove $u(x, t) \geq u_{n}(x)-\varepsilon$ and $v(x, t) \leq v_{n}(x)+\varepsilon$ for large $t$. Thus,

$$
\frac{\partial u}{\partial t}-\Delta u=u(a-u-c v) \leq u\left(a-u-c v_{n}-c \varepsilon\right)
$$

for large $t$. Now $u_{n+1}$ is the unique nontrivial nonnegative solution of

$$
-\Delta u=u\left(a-c v_{n}-u\right) \text {. }
$$

(As in [9], our assumptions ensure that $u_{n+1}$ exists.) By continuity, there is a unique nonnegative solution $u_{n+1}^{\varepsilon}$ of $-\Delta u=u\left(a-c v_{n}-c \varepsilon-u\right)$ close to $u_{n+1}$. Now every solution of

$$
\frac{\partial u}{\partial t}-\Delta u=u\left(a-u-c v_{n}-c \varepsilon\right)
$$

with nonnegative nontrivial initial-value approaches $u_{n+1}^{\varepsilon}$ in $C_{0}(\bar{\Omega})$ as $t \rightarrow$ $\infty$. Hence by comparing (19) with $(20)$ and by using the parabolic maximum principle, we see that $u(t) \geq u_{n+1}^{\varepsilon}-\delta$ for large $t$. Hence $u(t) \geq u_{n+1}-\varepsilon$ for large $t$. Remember that $u_{n+1}^{\varepsilon} \rightarrow u_{n+1}$ as $\varepsilon \rightarrow 0$. We can establish the corresponding estimate for $v$ (that is, $\left.v(t) \leq v_{n}+\varepsilon\right)$ provided that $r\left((-\Delta)^{-1}\left(d-e u_{n+1}\right)\right)>$ 1. This is the condition for $v_{n+1}$ to exist. In fact, $v_{n+1}$ is the nontrivial nonnegative solution of $-\Delta v=v\left(d-v-e u_{n+1}\right)$.

Thus we have proved our inductive step if the sequence $\left\{\left(u_{n}, v_{n}\right)\right\}$ does not terminate at $n$. Now suppose that $r\left((-\Delta)^{-1}\left(d-e u_{n+1}\right)\right) \leq 1$. Thus $v_{n+1}$ does 
not exist. By continuity, the maximal nonnegative solution $v_{n+1}^{\varepsilon}$ of $-\Delta v=$ $v\left(d-e u_{n+1}+e \varepsilon-v\right)$ either vanishes identically or is uniformly small. We can use the results for scalar equations, the parabolic maximum principle, and the estimates for $u_{n+1}$ to prove that $v(t) \leq v_{n+1}^{\varepsilon}+\varepsilon$ for large $t$ (cf. above). Thus $v(t) \rightarrow 0$ uniformly as $t \rightarrow \infty$. We can then use the first equation to prove that $u(t) \rightarrow \bar{u}$ as $t \rightarrow \infty$. Hence we see that if the sequence terminates, then $(u(t), v(t)) \rightarrow(\bar{u}, 0)$ as $t \rightarrow \infty$. This completes the proof of our claim.

There are two comments to be made on the above proof. First, with care, the smoothness assumption on $\partial \Omega$ can be avoided by working with $L^{\infty}$ and $L^{p}$ spaces. Second, we are using that, if $u_{0}$ and $v_{0}$ do not vanish identically, then neither $u(t)$ nor $v(t)$ vanish for $t>0$. This follows easily by obtaining a differential inequality for $\left\langle u(t), \phi_{1}\right\rangle$ where $\phi_{1}$ is the positive eigenfunction of $-\Delta$.

There is an alternative way of proving our result above in smooth domains. We sketch this. Since $c<\bar{c}$ (or more generally $(0, \bar{v})$ is unstable to perturbations in $\left.K_{2}\right)$, the 1-dimensional unstable manifold $U$ of $(1)$ at $(0, \bar{v})$ corresponding to the smallest real eigenvalue of the linearization at $(0, \bar{v})$ (or centre manifold if $c=\bar{c}$ ) has one part pointing into $D$ and one part pointing out of $D$. Denote by $U^{+}$the part pointing into $D$. If $c<\bar{c}$ or $c=\bar{c}$ and $(0, \bar{v})$ is unstable to perturbations in $K_{2}$, we easily see that the flow $\pi(t,(u, v))$ corresponding to (1) moves out along the one-dimensional manifold $U^{+}$. (Note that it is easy to prove that, for fixed $t, \pi(t,()$,$) is monotone for our order$ $\leq_{S}$. Using this, we can easily deduce that $(0, \bar{v})$ is stable to perturbations in $K_{2}$ if $(0, \bar{v})$ is stable on $U^{+}$.) Moreover, $U^{+}$is tangent to the positive eigenfunction of the linearization at $(0, \bar{v})$ which has both components positive. Since $\partial \Omega$ is smooth, we easily deduce that if $s, \tilde{t} \in U^{+}$and $s, \tilde{t}$ are close to $(0, \bar{v})$, then either $s \geq_{S} \tilde{t}$ or $s \leq_{S} \tilde{t}$. In particular, if $(x, y) \in U^{+}$ and is close to $(0, \bar{v})$, then $\pi(t,(x, y)) \geq_{S}(x, y)$ for small positive $t$. By the monotonicity of $\pi$, it follows that $\pi(t,(x, y))$ is increasing in $t$ for all $t$. However, $(x, y) \leq_{S}(\bar{u}, 0)$ if $(x, y)$ is close to $(0, \bar{v})$ on $U^{+}$and hence $\pi(t,(x, y)) \leq_{S} \pi(t,(\bar{u}, 0))=(\bar{u}, 0)$ for all $t>0$ by the monotonicity. Note that by the construction of $U^{+}$(cf. [20, $\left.\S 6.2\right]$ ), we see that many norms induce equivalent metrics on $U^{+}$. Thus, if $(x, y)$ is close to $(0, \bar{v})$ in $L^{\infty}$, it is close in $C^{1}$. Hence $\pi(t,(x, y))$ increases to a solution $(\tilde{u}, \tilde{v})$ of (2). Since any solution of $(2)$ is greater than or equal to $(0, \bar{v})$ (for our order), it follows easily from the monotonicity and smoothness that $(\tilde{u}, \tilde{v})$ is the minimal strictly positive solution of (2). Moreover, by the beginning of the proof of the first method, for any nontrivial nonnegative initial value $\left(u_{0}, v_{0}\right)$ with neither component vanishing identically, $\omega\left(u_{0}, v_{0}\right) \geq_{S}(0, \bar{v})$. Hence by the monotonicity, one can deduce that $\omega\left(u_{0}, v_{0}\right) \geq_{s}(x, y)$ if $(x, y)$ is close to $(0, \bar{v})$ in $U^{+}$. (Here one uses that $\pi$ has the stronger monotonicity property that if $(x, y)>_{S}\left(x_{1}, y_{1}\right)$ then $\pi(t,(x, y))-\pi\left(t,\left(x_{1}, y_{1}\right)\right)$ is interior to the cone for $t>0$ if we work with $C^{1}$ spaces. Here we use similar ideas to [22 
or 26].) Hence, by the monotonicity of $\pi, \omega\left(u_{0}, v_{0}\right) \geq_{S}(\tilde{u}, \tilde{v})$ as required. It can be shown that $(0, \bar{v})$ is unstable to perturbation in $K_{2}$ (unstable for the flow) is equivalent to the conditions that $(0, \bar{v})$ have nonzero index in $D$ (for the map $A$ ).

The proof in the last paragraph has the advantage that it sometimes applies when $c=\bar{c}$ but it seems to need rather more smoothness on $\partial \Omega$. However, it is conceptally nicer. The first method has the advantage that it can be used to limit $\omega$ limit sets for systems without monotonicity (for example, predator prey equations). Note that either method implies that $(\tilde{u}, \tilde{v})$ has the same symmetries as $\Omega$ and is constant for Neumann boundary conditions. Either method also implies that, if $c<\bar{c}$ and $e<\bar{e}$ and there is a unique strictly positive solution $(\tilde{u}, \tilde{v})$, then all solutions with initial values $\left(u_{0}, v_{0}\right)$ nonnegative and neither $u_{0}$ nor $v_{0}$ vanishing identically approach $(\tilde{u}, \tilde{v})$ as $t \rightarrow \infty$. This method can be used to give an alternative proof of the main result of [3].

The most interesting question is whether the $\omega$ limit set $\omega\left(\left(u_{0}, v_{0}\right)\right)$ need only be made up of stationary solutions. This does not seem obvious even for Neumann boundary conditions (at least when $c>\bar{c}$ and $e>\bar{e}$ ). Note that for the predator prey model with Neumann boundary conditions and an "asocial" nonlinearity time-periodic solutions can bifurcate off the constant solutions.

\section{REFERENCES}

1. H. Amann, Fixed point equations and nonlinear eigenvalue problems in ordered Banach spaces, SIAM Rev. 18 (1976), 620-709.

2. H. Berestycki and P. L. Lions, Some applications of the method of sub-and super-solutions, in Bifurcation and Nonlinear Eigenvalue Problems, Lecture Notes in Math., vol. 782, SpringerVerlag, Berlin, 1980, pp. 16-41.

3. P. N. Brown, Decay to uniform states in ecological interactions, SIAM J. Appl. Math. 38 (1980), 22-37.

4. R. Cantrell and C. Cosner, On the steady-state problem for the Volterra-Lotka competition model with diffusion, Houston J. Math. 13 (1987), 337-352.

5. E. Conway, Diffusion and the predator prey interaction: steady state with flux at the boundaries, in Nonlinear Partial Differential Equations (J. Smoller, ed.), Amer. Math. Soc., Providence, R.I., 1983, pp. 215-234.

6. C. Cosner and A. Lazer, Stable coexistence states in the Volterra Lotka competition model with diffusion, SIAM J. Appl. Math. 44 (1984), 1112-1132.

7. M. Crandall and P. H. Rabinowitz, Birfucation, perturbation of simple eigenvalues and linearized stability, Arch. Rational Mech. Anal. 52 (1973), 161-180.

8. E. N. Dancer, On positive solutions of some pairs of differential equations, Trans. Amer. Math. Soc. 284 (1984), 729-743.

9. __ On positive solutions of some pairs of differential equations. II, J. Differential Equations 60 (1985), 236-258.

10. __ Multiple fixed points of positive maps, J. Reine Angew Math. 371 (1986), 46-66.

11. _ On the indices of fixed points of mappings in cones and applications, J. Math. Anal. Appl. 91 (1983), 131-151.

12. __ On the number of positive solutions of weakly nonlinear elliptic equations when a parameter is large, Proc. London Math. Soc. (3) 53 (1986, 429-452. 
13. _ Counterexamples to some conjectures on the number of solutions of nonlinear equations, Math. Ann. 272 (1985), 421-440.

14. _ . The effect of domain shape on the number of positive solutions of certain nonlinear equations, J. Differential Equations 74 (1988), 120-156.

15. $\ldots$, A note on an equation with critical exponent, Bull. London Math. Soc. 20 (1988), 600-602.

16. E. N. Dancer and P. Hess, On stable solutions of quasilinear periodic parabolic problems, Ann. Scuola Norm. Sup. Pisa Cl. Sci. (4) 14 (1987), 123-141.

17. H. Federer, Geometric measure theory, Springer, Berlin, 1969.

18. D. Gilbarg and N. Trudinger, Elliptic partial differential equations of second order, Springer, Berlin, 1977.

19. A. Granas, Points fixes pour less applications compact, Univ. of Montreal Press, Montreal, 1980.

20. D. Henry, Geometric theory of semilinear parabolic equations, Springer, Berlin, 1981.

21. E. Hewitt and K. Stromberg, Real and abstract analysis, Springer, Berlin, 1965.

22. M. W. Hirsch, Differential equations and convergence almost everywhere in monotone flows, in Nonlinear Partial Differential Equations (J. Smoller, ed.), Amer. Math. Soc., Providence, R.I., 1983, pp. 265-285.

23. T. Kato, Perturbation theory for linear operators, Springer, Berlin, 1975.

24. P. Korman and A. Leung, On the existence and uniqueness of positive steady states in the Volterra-Lotka ecological models with diffusion, Appl. Anal. 26 (1987), 145-160.

25. O. Ladyzhenskaya and N. Uraltseva, Linear and quasilinear elliptic equations, Academic Press, New York, 1970.

26. H. Matano, Existence of non-trivial unstable sets for equilibriums of strongly order-preserving systems, J. Fac. Sci. Univ. Tokyo Sect. IA Math. 30 (1984), 645-673.

27. R. Nussbaum, The fixed point index for locally condensing maps, Ann. Mat. Pura Appl. 87 (1971), 217-258.

28. M. Protter and H. Weinberger, Maximum principles in differential equations, Prentice-Hall, Englewood Cliffs, N.J., 1967.

29. H. H. Schaefer, Topological vector spaces, Macmillan, New York, 1966.

Department of Mathematics, Statistics and Computing Science, The University of New England, Armidale, New South Wales 2351, Australia 